\title{
Differential Invariant Algebras
}

\author{
Peter J. Olver ${ }^{\dagger}$ \\ School of Mathematics \\ University of Minnesota \\ Minneapolis, MN 55455 \\ olver@umn.edu \\ http://www . math. umn.edu/ olver
}

\begin{abstract}
The equivariant method of moving frames provides a systematic, algorithmic procedure for determining and analyzing the structure of algebras of differential invariants for both finite-dimensional Lie groups and infinite-dimensional Lie pseudo-groups. This paper surveys recent developments, including a few surprises and several open questions.
\end{abstract}

\section{Introduction.}

Differential invariants are the fundamental building blocks for constructing invariant differential equations and invariant variational problems, as well as determining their explicit solutions and conservation laws. The equivalence, symmetry and rigidity properties of submanifolds are all governed by their differential invariants. Additional applications abound in differential geometry and relativity, classical invariant theory, computer vision, integrable systems, geometric flows, calculus of variations, geometric numerical integration, and a host of other fields of both pure and applied mathematics.

$\dagger$ Supported in part by NSF Grant DMS 11-08894.

November 16, 2018 
Therefore, the underlying structure of the algebras ${ }^{\ddagger}$ of differential invariants for a broad range of transformation groups becomes a topic of great importance. Until recently, though, beyond the relatively easy case of curves, i.e., functions of a single independent variable, and a few explicit calculations for individual higher dimensional examples, surprisingly little was known concerning either the structure theory or general computational algorithms. In my second book, [33], I surveyed the state of the art in the mid 1990's, and suggested adapting tools from commutative algebra in this endeavor. While I was not anticipating significant progress in the near term, it happened that a few years later, with the vital assistance of Mark Fels, who was visiting Minnesota as a Canadian NSERC postdoc, a new, equivariant reformulation of the Cartan method of moving frames was proposed in $[\mathbf{9}]$. This theory had the great advantage that, while the key ideas are remarkably elementary, they quickly lead to powerful algorithms for constructing and classifying invariant quantities associated with Lie group actions, including differential invariants, invariant differential operators, invariant differential forms, joint and joint differential invariants, invariant tensors, invariant differential equations, invariant variational problems, invariant numerical algorithms, and so on. Moreover, the resulting "recurrence relations" serve to completely prescribe the underlying structure of the algebra of differential invariants, and, more generally, invariant differential forms. Even more amazingly, this differential algebraic structure can be determined $a b$ initio, requiring only the formulas for the infinitesimal generators of the group action along with some linear differential algebra, and without and need for the explicit formulas for the differential invariants, the moving frame, or even the group transformations! The recent book by Mansfield, [27], provides a very readable account of the basic ideas. Subsequently, in collaboration with Juha Pohjanpelto, $[\mathbf{4 4}, \mathbf{4 5}]$, the equivariant moving frame construction was successfully extended to encompass infinite-dimensional pseudo-groups. The methods remain completely algorithmic, but are necessarily more intricate owing to the lack of a general abstract object that can adequately represent a Lie pseudo-group.

In this paper, I will concentrate on the applications of moving frames to the study of differential invariant algebras, for both finite-dimensional and infinite-dimensional group actions, and review some recent, surprising developments. Open problems and future research directions are indicated along the way. Additional applications and developments in the theory can be found in the cited references; see, in particular, [41], for a recent survey of the finite-dimensional case.

\section{Moving Frames and Differential Invariants.}

Let $G$ denote either a finite-dimensional Lie group or, more generally, an infinitedimensional Lie pseudo-group that acts (locally) on an $m$-dimensional manifold $M$. Here, all manifolds, functions, groups, etc., are assumed to be smooth, i.e., $\mathrm{C}^{\infty}$. We are interested in the induced action of $G$ on $p$-dimensional submanifolds $N \subset M$. For each integer $0 \leq n \leq \infty$, let $\mathrm{J}^{n}=\mathrm{J}^{n}(M, p)$ denote the $n^{\text {th }}$ order submanifold jet bundle, defined as the set of equivalence classes under the equivalence relation of $n^{\text {th }}$ order contact at a single

$\ddagger$ Here, we use the term "algebra" loosely — see below for details. 
point; see [32; Chapter 3] for details. For $k \geq n$, we use $\pi_{n}^{k}: \mathrm{J}^{k} \rightarrow \mathrm{J}^{n}$ to denote the natural projection. Since $G$ preserves the contact equivalence relation, it induces an action on $\mathrm{J}^{n}$, known as its $n^{\text {th }}$ order prolongation. The formulas for the prolonged group action are readily found by implicit differentiation.

A real-valued function ${ }^{\dagger} I: \mathrm{J}^{n} \rightarrow \mathbb{R}$ is known as a differential invariant if it is unaffected by the prolonged group transformations, so $I\left(g^{(n)} \cdot z^{(n)}\right)=I\left(z^{(n)}\right)$ for all $z^{(n)} \in \mathrm{J}^{n}$ and all $g \in G$ such that both $z^{(n)}$ and $g^{(n)} \cdot z^{(n)}$ lie in the domain of $I$. Clearly, any functional combination of differential invariants is a differential invariant (on the common domain of definition) and thus we speak, somewhat loosely, of the algebra of differential invariants associated with the action of the transformation group on submanifolds of a specified dimension. Since differential invariants are often only locally defined ${ }^{\ddagger}$, we should introduce the category of sheaves of differential invariants, [21]. However, for our local results, this extra level of abstraction is unnecessary, and so we will leave their sheaf-theoretic reformulation as a simple translational exercise for the experts.

Any finite-dimensional group action admits an infinite number of functionally independent differential invariants of progressively higher and higher order. On the other hand, infinite-dimensional pseudo-groups may or may not admit nontrivial differential invariants. For example, both the pseudo-group of all local diffeomorphisms, or that of all local symplectomorphisms, [29], act transitively on a dense open subset of the jet space, and hence admit no non-constant local invariants. (Global invariants, such as Gromov's symplectic capacity, [11], are not, at least as far as I know, amenable to moving frame techniques.)

The Fundamental Basis Theorem states that the entire algebra of differential invariants can be generated from a finite number of low order invariants by repeated invariant differentiation. In differential invariant theory, it assumes the role played by the algebraic Hilbert Basis Theorem for polynomial ideals, [8]. The technical "eventually free" requirement on pseudo-groups will be explained later - see Definition 6.6 and the ensuing remarks. Extending the Basis Theorem to non-free pseudo-group actions is a significant open problem.

Theorem 2.1. Let $G$ be a finite-dimensional Lie group or, more generally, a Lie pseudo-group acting eventually freely on jets of $p$-dimensional submanifolds $N \subset M$. Then, locally, there exist a finite collection of generating differential invariants $\mathcal{I}=\left\{I_{1}, \ldots, I_{\ell}\right\}$, along with exactly $p$ invariant differential operators $\mathcal{D}_{1}, \ldots, \mathcal{D}_{p}$, such that every differential invariant can be locally expressed as a function of the generating invariants and their invariant derivatives $\mathcal{D}_{J} I_{\sigma}=\mathcal{D}_{j_{1}} \mathcal{D}_{j_{2}} \cdots \mathcal{D}_{j_{k}} I_{\sigma}$, for $\sigma=1, \ldots, l, 1 \leq j_{\nu} \leq p, k=\# J \geq 0$.

The Basis Theorem was first formulated by Lie, [23; p. 760], for finite-dimensional group actions. Modern proofs of Lie's result can be found in $[\mathbf{3 3}, \mathbf{4 7}]$. The theorem was extended to infinite-dimensional pseudo-groups by Tresse, [51]. A rigorous version,

$\dagger$ Throughout, functions, maps, etc., may only be defined on an open subset of their indicated source space: $\operatorname{dom} I \subset \mathrm{J}^{n}$.

$\ddagger$ On the other hand, in practical examples, differential invariants turn out to be algebraic functions defined on Zariski open subsets of jet space, and so reformulating the theory in a more algebro-geometric framework would be a worthwhile endeavor; see, for instance, [15]. 
based on the machinery of Spencer cohomology, was established by Kumpera, [21]. A recent generalization to pseudo-group actions on differential equations (submanifolds of jet space) can be found in [20], while [30] introduces an approach based on Weil algebras. A constructive proof of the pseudo-group version, based on the moving frame machinery, first appeared in $[\mathbf{4 5}]$.

The invariant differential operators map differential invariants to differential invariants. They do not necessarily commute, and so the order of differentiation is important. However, each commutator can be re-expressed as a linear combination thereof,

$$
\left[\mathcal{D}_{j}, \mathcal{D}_{k}\right]=\mathcal{D}_{j} \mathcal{D}_{k}-\mathcal{D}_{k} \mathcal{D}_{j}=\sum_{i=1}^{p} Y_{j k}^{i} \mathcal{D}_{i}
$$

where the coefficients $Y_{j k}^{i}=-Y_{k j}^{i}$ are themselves differential invariants, known as the commutator invariants.

Furthermore, the differentiated invariants $\mathcal{D}_{J} I_{\sigma}$ are not necessarily functionally independent, but may be subject to certain functional relations or differential syzygies of the form

$$
H\left(\ldots \mathcal{D}_{J} I_{\sigma} \ldots\right) \equiv 0 .
$$

The Syzygy Theorem, first stated (not quite correctly) in [9] for finite-dimensional actions, and then rigorously formulated and proved in [45], states that there are a finite number of generating differential syzygies. Again, this is by way of analogy with the Hilbert Syzygy Theorem for polynomial ideals, [8]. See Theorem 6.12 for a precise statement of the differential invariant version.

A very familiar, elementary example is the (special) Euclidean group $G=\mathrm{SE}(3)=$ $\mathrm{SO}(3) \ltimes \mathbb{R}^{3}$, consisting of all orientation-preserving isometries of $M=\mathbb{R}^{3}$. When $G$ acts on space curves — one-dimensional submanifolds — the differential invariant algebra is generated by the curvature $\kappa$, torsion $\tau$, and their successive derivatives with respect to arc length. Similarly, the differential invariants for the action of $\operatorname{SE}(3)$ on surfaces $S \subset \mathbb{R}^{3}$ consist of the Gauss and mean curvatures and their derivatives with respect to the two non-commuting invariant differential operators provided by the diagonalizing Frenet frame on the surface. In this case, there is a single fundamental differential syzygy among the curvature invariants: the Gauss-Codazzi formula. See Guggenheimer's book, [12], for the classical moving frame treatment of these (and many other) examples. The equivariant approach, which is more straightforward to formulate and to implement, as well as being completely algorithmic, will be presented below.

\section{Equivariant Moving Frames.}

Inspired by Cartan's remarkable development and application, $[\mathbf{6}, \mathbf{1 2}]$, of a method that dates back to 1800 , cf. [1], the equivariant approach to the method of moving frames was first fully formulated in $[\mathbf{9}]$, and then extended to infinite-dimensional pseudo-groups in $[44]$. In this section, we restrict our attention to the simpler finite-dimensional Lie group version, and so, until we get to Section $6, G$ will denote an $r$-dimensional Lie group acting smoothly on an $m$-dimensional manifold $M$. 
Definition 3.1. A moving frame is a $G$-equivariant map $\rho: M \rightarrow G$.

There are two principal types of equivariance:

$$
\rho(g \cdot z)= \begin{cases}g \cdot \rho(z), & \text { left moving frame, } \\ \rho(z) \cdot g^{-1}, & \text { right moving frame. }\end{cases}
$$

In classical geometries, one can always reinterpret the usual frame-based moving frame, cf. [12], as a left-equivariant map. On the other hand, right equivariant moving frames are often easier to compute, and will be the primary focus here. Bear in mind that if $\rho(z)$ is a right-equivariant moving frame, then $\widehat{\rho}(z)=\rho(z)^{-1}$ is a left-equivariant counterpart.

It is not difficult to establish the basic requirements for the existence of an equivariant moving frame, $[\mathbf{9}]$.

Theorem 3.2. A moving frame exists in a neighborhood of a point $z \in M$ if and only if $G$ acts freely and regularly near $z$.

Recall that $G$ acts freely if the isotropy subgroup $G_{z}=\{g \in G \mid g \cdot z=z\}$ of each point $z \in M$ is trivial: $G_{z}=\{e\}$. This implies local freeness, meaning that the isotropy subgroups $G_{z}$ are all discrete, or, equivalently, that the orbits all have the same dimension, $r$, as $G$ itself. In practice, local freeness will suffice to establish a locally equivariant moving frame. Regularity requires that, in addition, the orbits form a regular foliation; it is a global condition that plays no role in practical applications.

The explicit construction of a moving frame relies on the choice of a (local) crosssection to the group orbits, meaning an $(m-r)$-dimensional submanifold $K \subset M$ that intersects each orbit transversally and at most once.

Theorem 3.3. Let $G$ act freely and regularly on $M$, and let $K \subset M$ be a crosssection. Given $z \in M$, let $g=\rho(z)$ be the unique group element that maps $z$ to the cross-section: $g \cdot z=\rho(z) \cdot z \in K$. Then $\rho: M \rightarrow G$ is a right moving frame.

Given local coordinates $z=\left(z_{1}, \ldots, z_{m}\right)$ on $M$, suppose the cross-section $K$ is defined by the $r$ equations

$$
Z_{1}(z)=c_{1}, \quad \ldots \quad Z_{r}(z)=c_{r},
$$

where $Z_{1}, \ldots, Z_{r}$ are scalar-valued functions, while $c_{1}, \ldots, c_{r}$ are suitably chosen constants. In many applications, the $Z_{\sigma}$ are merely a subset of the coordinate functions $z^{1}, \ldots, z^{m}$, in which case they are said to define a coordinate cross-section. In general, the associated right moving frame $g=\rho(z)$ is obtained by solving the normalization equations

$$
Z_{1}(g \cdot z)=c_{1}, \quad \ldots \quad Z_{r}(g \cdot z)=c_{r},
$$

for the group parameters $g=\left(g_{1}, \ldots, g_{r}\right)$ in terms of the coordinates $z=\left(z_{1}, \ldots, z_{m}\right)$. Transversality combined with the Implicit Function Theorem implies the existence of a local solution to these algebraic equations. In most applications, the art of the method is to select a cross-section that simplifies the calculations as much as possible.

Once a moving frame is specified through the choice of a cross-section, it induces a canonical invariantization process that maps functions to invariants. Invariantization (and its consequences) provides the preeminent advantage of the equivariant approach over other moving frame theories. 
Definition 3.4. The invariantization of a function $F: M \rightarrow \mathbb{R}$ is the the unique invariant function $I=\iota(F)$ that agrees with $F$ on the cross-section: $I|K=F| K$.

In practice, the invariantization of a function $F(z)$ is obtained by first transforming it according to the group, $F(g \cdot z)$, and then replacing all the group parameters by their moving frame formulae $g=\rho(z)$, so that $\iota[F(z)]=F(\rho(z) \cdot z)$. In particular, invariantization of the coordinate functions yields the fundamental invariants: $I_{j}(z)=\iota\left(z_{j}\right), j=1, \ldots, m$. Once these have been computed, the invariantization of a general function $F(z)$ is simply given by

$$
\iota\left[F\left(z_{1}, \ldots, z_{m}\right)\right]=F\left(I_{1}(z), \ldots, I_{m}(z)\right) .
$$

In particular, the functions defining the cross-section (3.2) have constant invariantization, $\iota\left(Z_{\sigma}(z)\right)=c_{\sigma}$, and are known as the phantom invariants, leaving precisely $m-r$ functionally independent basic invariants. Moreover, if $J\left(z_{1}, \ldots, z_{m}\right)$ is any invariant, then clearly $\iota(J)=J$, which implies the elegant and powerful Replacement Rule

$$
J\left(z_{1}, \ldots, z_{m}\right)=J\left(I_{1}(z), \ldots, I_{m}(z)\right),
$$

that can be used to immediately rewrite $J$ in terms of the basic invariants.

Of course, most interesting group actions are not free, and therefore do not admit moving frames in the sense of Definition 3.1. There are two classical methods that (usually) convert a non-free action into a free action. The first is to look at the Cartesian product action of $G$ on several copies of $M$, leading to joint invariants, [36]. The second is to prolong the group action to jet space, which is the natural setting for the traditional moving frame theory, and leads to differential invariants, $[\mathbf{9}]$. Combining the two methods of jet prolongation and Cartesian product results in joint differential invariants, [36]. In applications of symmetry methods to numerical analysis, one requires an amalgamation of all these actions into a common framework, called multispace, [37]. In this paper we will only treat the jet space mode of prolongation, and refer the interested reader to [41] for a recent survey of other developments.

We can assume, without loss of generality, that $G$ acts effectively on open subsets of $M$, meaning that the only group element that fixes every point in any open $U \subset M$ is the identity element. This implies, [35], that the prolonged action is locally free on a dense open subset $V^{n} \subset \mathrm{J}^{n}$ for $n$ sufficiently large. In fact, in all known examples, the prolonged action is, in fact, free on an open subset of $\mathrm{J}^{n}$ for $n \gg 0$, although there is, frustratingly, no general proof or counterexample known as yet. The points $z^{(n)} \in V^{n}$ are called regular jets.

As above, the normalization construction based on a choice of local cross-section $K^{n} \subset V^{n}$ to the prolonged group orbits can be used to produce an $n^{\text {th }}$ order equivariant moving frame $\rho: \mathrm{J}^{n} \rightarrow G$ in a neighborhood of any regular jet. The cross-section $K^{n}$ is prescribed by a collection of $r=\operatorname{dim} G$ independent $n^{\text {th }}$ order differential functions

$$
Z_{1}\left(z^{(n)}\right)=c_{1}, \quad \ldots \quad Z_{r}\left(z^{(n)}\right)=c_{r} .
$$

As before, the associated right moving frame $g=\rho\left(z^{(n)}\right)$ is obtained by solving the normalization equations

$$
Z_{1}\left(g^{(n)} \cdot z^{(n)}\right)=c_{1}, \quad \ldots \quad Z_{r}\left(g^{(n)} \cdot z^{(n)}\right)=c_{r},
$$


for the group parameters $g=\left(g_{1}, \ldots, g_{r}\right)$ in terms of the jet coordinates $z^{(n)}$. Once the moving frame is established, the induced invariantization process will map general differential functions $F\left(z^{(k)}\right)$ to differential invariants $I=\iota(F)$, which are obtained by substituting the moving frame formulas for the group parameters in their transformed version: $I\left(z^{(k)}\right)=\left.F\left(g^{(k)} \cdot z^{(k)}\right)\right|_{g=\rho\left(z^{(n)}\right)}$.

For calculations, we introduce local coordinates $z=(x, u)$ on $M$, considering the first $p$ components $x=\left(x^{1}, \ldots, x^{p}\right)$ as independent variables, and the latter $q=m-p$ components $u=\left(u^{1}, \ldots, u^{q}\right)$ as dependent variables. These induce local coordinates $z^{(n)}=\left(x, u^{(n)}\right)$ on $\mathrm{J}^{n}$ with components $u_{J}^{\alpha}$ representing the partial derivatives of the dependent variables with respect to the independent variables, $[\mathbf{3 2}, 33]$. Submanifolds whose jets can be coordinatized by $\left(x, u^{(n)}\right)$ are those that are transverse to the vertical fibers $\{x=$ const. $\}$, or, equivalently, those that can be locally identified as the graph of a function $u=f(x)$. The fundamental differential invariants are obtained by invariantization of the coordinate functions.

$$
H^{i}=\iota\left(x^{i}\right), \quad I_{J}^{\alpha}=\iota\left(u_{J}^{\alpha}\right), \quad \alpha=1, \ldots, q, \quad \# J \geq 0,
$$

and we abbreviate $\left(H, I^{(k)}\right)=\iota\left(x, u^{(k)}\right)$ for those obtained from the jet coordinates of order $\leq k$. Keep in mind that the invariant $I_{J}^{\alpha}$ has order $\leq \max \{\# J, n\}$, where $n$ is the order of the moving frame. The fundamental differential invariants (3.8) naturally split into two classes: The $r=\operatorname{dim} G$ combinations defining the cross-section will be constant, and are known as the phantom differential invariants. The remainder, called the basic differential invariants, form a complete system of functionally independent differential invariants.

According to (3.4), the invariantization process can be simply implemented by replacing each jet coordinate by the corresponding normalized differential invariant (3.8):

$$
\iota\left[F\left(x, u^{(k)}\right)\right]=F\left(H, I^{(k)}\right) .
$$

In particular, the Replacement Rule, cf. (3.5), allows one to straightforwardly rewrite any differential invariant in terms the basic invariants:

$$
J\left(x, u^{(k)}\right)=J\left(H, I^{(k)}\right) \quad \text { whenever } J \text { is a differential invariant. }
$$

The specification of independent and dependent variables on $M$ splits the differential one-forms on the submanifold jet space $\mathrm{J}^{\infty}$ into horizontal forms, spanned by $d x^{1}, \ldots, d x^{p}$, and contact forms, spanned by the basic contact forms

$$
\theta_{J}^{\alpha}=d u_{J}^{\alpha}-\sum_{i=1}^{p} u_{J, i}^{\alpha} d x^{i}, \quad \alpha=1, \ldots, q, \quad 0 \leq \# J .
$$

In general, a differential form $\theta$ is called a contact form if and only if it is annihilated by all jets, so $\theta \mid \mathrm{j}_{\infty} S=0$ for all $p$-dimensional submanifolds $S \subset M$. We let $\pi_{H}$ and $\pi_{V}$ be the projections onto horizontal and contact components, respectively. Further, the differential on $\mathrm{J}^{\infty}$ splits into horizontal and vertical components: $d=d_{H}+d_{V}$. Closure, $d \circ d=0$, implies that $d_{H} \circ d_{H}=0=d_{V} \circ d_{V}$, while $d_{H} \circ d_{V}=-d_{V} \circ d_{H}$. The

$\dagger$ The splitting only works at infinite order. 
resulting structure is known as the variational bicomplex, $[\mathbf{4}, \mathbf{1 8}]$, and lies at the heart of the geometric approach to differential equations, variational problems, symmetries and conservation laws, characteristic classes, etc.

The invariantization process induced by a moving frame can also be applied to differential forms on jet space. Thus, given a differential form $\omega$, its invariantization $\iota(\omega)$ is the unique invariant differential form that agrees with $\omega$ on the cross-section. As with differential functions, the invariantized form is found by first transforming (pulling back) the form by the prolonged group action, and then replacing the group parameters by their moving frame formulae:

$$
\iota(\omega)=\left.\left(g^{(k)}\right)^{*}(\omega)\right|_{g=\rho\left(z^{(n)}\right)} .
$$

An invariantized contact form remains a contact form, while an invariantized horizontal form is, in general, a combination of horizontal and contact forms. The complete collection of invariantized differential forms serves to define the invariant variational bicomplex, studied in detail in [18].

For the purposes of analyzing the differential invariants, we can ignore the contact forms. (Although they are important if one is interested in invariant variational problems, [18], or submanifold flows, [39].) The horizontal components of the invariantized horizontal forms

$$
\omega^{i}=\pi_{H}\left(\varpi^{i}\right)=\pi_{H}\left[\iota\left(d x^{i}\right)\right], \quad i=1, \ldots, p .
$$

form, in the language of [33], a contact-invariant coframe. The corresponding dual invariant differential operators $\mathcal{D}_{1}, \ldots, \mathcal{D}_{p}$ are defined by

$$
d_{H} F=\sum_{i=1}^{p}\left(D_{i} F\right) d x^{i}=\sum_{i=1}^{p}\left(\mathcal{D}_{i} F\right) \omega^{i}
$$

for any differential function $F\left(x, u^{(k)}\right)$. Here $D_{1}, \ldots, D_{p}$ are the usual total derivative operators, [32].

Example 3.5. The paradigmatic example is the action of the Euclidean group $\mathrm{SE}(2)=\mathrm{SO}(2) \ltimes \mathbb{R}^{2}$ on plane curves $C \subset M=\mathbb{R}^{2}$. A group transformation maps the point $z=(x, u)$ to the point $w=(y, v)=g \cdot z$, given by

$$
y=x \cos \phi-u \sin \phi+a, \quad v=x \sin \phi+u \cos \phi+b,
$$

where $g=(\phi, a, b)$ are the group parameters. The prolonged group transformations are obtained via implicit differentiation:

$$
v_{y}=\frac{\sin \phi+u_{x} \cos \phi}{\cos \phi-u_{x} \sin \phi}, \quad v_{y y}=\frac{u_{x x}}{\left(\cos \phi-u_{x} \sin \phi\right)^{3}}, \quad \text { and so on. }
$$

Observe that the prolonged action is locally free on the first order jet space $\mathrm{J}^{1}$, and free on the open subset $\left\{u_{x x} \neq 0\right\} \subset \mathrm{J}^{2}$.

The classical (locally equivariant) moving frame is based on the cross-section

$$
K^{1}=\left\{x=u=u_{x}=0\right\} \subset \mathrm{J}^{1} .
$$


Solving the corresponding normalization equations $y=v=v_{y}=0$ for the group parameters produces the right moving frame

$$
\phi=-\tan ^{-1} u_{x}, \quad a=-\frac{x+u u_{x}}{\sqrt{1+u_{x}^{2}}}, \quad b=\frac{x u_{x}-u}{\sqrt{1+u_{x}^{2}}} .
$$

Invariantization of the jet coordinate functions is accomplished by substituting the moving frame formulae (3.18) into the prolonged group transformations (3.16), producing the fundamental differential invariants:

$$
\begin{aligned}
& H=\iota(x)=0, \quad I_{0}=\iota(u)=0, \quad I_{1}=\iota\left(u_{x}\right)=0 \\
& I_{2}=\iota\left(u_{x x}\right)=\frac{u_{x x}}{\left(1+u_{x}^{2}\right)^{3 / 2}}, \quad I_{3}=\iota\left(u_{x x x}\right)=\frac{\left(1+u_{x}^{2}\right) u_{x x x}-3 u_{x} u_{x x}^{2}}{\left(1+u_{x}^{2}\right)^{3}}
\end{aligned}
$$

and so on. The first three, corresponding to functions defining the the cross-section (3.17), are the phantom invariants. The lowest order basic differential invariant is the Euclidean curvature: $I_{2}=\kappa$. The higher order differential invariants will be identified below.

Similarly, to invariantize the horizontal form $d x$, we first apply a group transformation

$$
d y=\cos \phi d x-\sin \phi d u=\left(\cos \phi-u_{x} \sin \phi\right) d x-(\sin \phi) \theta,
$$

where $\theta=d u-u_{x} d x$ is the order zero basis contact form. Substituting the moving frame formulae (3.18) produces the invariant one-form

$$
\varpi=\iota(d x)=\sqrt{1+u_{x}^{2}} d x+\frac{u_{x}}{\sqrt{1+u_{x}^{2}}} \theta .
$$

Its horizontal component

$$
\omega=\pi_{H}(\varpi)=\sqrt{1+u_{x}^{2}} d x
$$

is the usual arc length element, and is invariant modulo contact forms. Thus, the dual invariant differential operator is the arc length derivative

$$
\mathcal{D}=\frac{1}{\sqrt{1+u_{x}^{2}}} D_{x}
$$

\section{Recurrence.}

In general, invariantization and differentiation do not commute. By a recurrence relation, we mean a formula that expresses a differentiated invariant in terms of the basic differential invariants. The recurrence relations are the master key that unlocks the entire structure of the algebra of differential invariants, including the specification of generators and the classification of syzygies. Remarkably, they can be explicitly determined without knowing the actual formulas for either the differential invariants, or the invariant differential operators, or even the moving frame! Indeed, they follow directly, using only linear algebra, from the formulas for the prolonged infinitesimal generators for the group action, combined with the specification of the cross-section normalizations. 
Let $\mathbf{v}_{1}, \ldots, \mathbf{v}_{r}$ be a basis for the infinitesimal generators of our $r$-dimensional transformation group, which we identify with a basis of its Lie algebra $\mathfrak{g}$. We prolong each infinitesimal generator to $\mathrm{J}^{n}$, resulting in the vector fields

$$
\mathbf{v}_{\kappa}^{(n)}=\sum_{i=1}^{p} \xi_{\kappa}^{i}(x, u) \frac{\partial}{\partial x^{i}}+\sum_{\alpha=1}^{q} \sum_{k=\# J=0}^{n} \varphi_{J, \kappa}^{\alpha}\left(x, u^{(k)}\right) \frac{\partial}{\partial u_{J}^{\alpha}}, \quad \kappa=1, \ldots, r .
$$

The coefficients $\varphi_{J, \kappa}^{\alpha}=\mathbf{v}_{\kappa}^{(n)}\left(u_{J}^{\alpha}\right)$ are provided by the well-known prolongation formula, $[\mathbf{3 2}, \mathbf{3 3}]$, first written in the following explicit non-recursive form in [31]:

$$
\varphi_{J, \kappa}^{\alpha}=D_{J}\left(\varphi_{\kappa}^{\alpha}-\sum_{i=1}^{p} \xi^{i} u_{i}^{\alpha}\right)+\sum_{i=1}^{p} \xi_{\kappa}^{i} u_{J, i}^{\alpha}
$$

in which $D_{J}=D_{j_{1}} \cdots D_{j_{k}}$ indicate iterated total derivative operators, and $u_{i}^{\alpha}=\partial u^{\alpha} / \partial x^{i}$.

Given a moving frame on jet space, the universal recurrence relation for differential invariants takes the following form.

Theorem 4.1. Let $F\left(x, u^{(n)}\right)$ be a differential function and $\iota(F)$ its moving frame invariantization. Then

$$
\mathcal{D}_{i}[\iota(F)]=\iota\left[D_{i}(F)\right]+\sum_{k=1}^{p} R_{i}^{\kappa} \iota\left[\mathbf{v}_{\kappa}^{(n)}(F)\right]
$$

where

$$
\mathcal{R}=\left\{R_{i}^{\kappa} \mid i=1, \ldots, p, \kappa=1, \ldots, r\right\}
$$

are known as the Maurer-Cartan differential invariants.

The Maurer-Cartan invariants can, in fact, be characterized as the coefficients of the horizontal components of the pull-backs of the Maurer-Cartan forms via the moving frame, [9]. Explicitly, suppose $\mu^{1}, \ldots, \mu^{r} \in \mathfrak{g}^{*}$ are the basis for the right-invariant Maurer-Cartan forms that is dual to the Lie algebra basis $\mathbf{v}_{1}, \ldots, \mathbf{v}_{r} \in \mathfrak{g}$. Then the horizontal components of their pull-backs $\nu^{\kappa}=\rho^{*} \mu^{\kappa}$ to $\mathrm{J}^{n}$ via the moving frame $\rho: \mathrm{J}^{n} \rightarrow G$ can be expressed as a linear combination of the contact-invariant coframe $\omega^{1}, \ldots, \omega^{p}$,

$$
\gamma^{\kappa}=\pi_{H}\left(\nu^{\kappa}\right)=\sum_{i=1}^{p} R_{i}^{\kappa} \omega^{i}, \quad \kappa=1, \ldots, r,
$$

whose coefficients $R_{i}^{\kappa}$ are the Maurer-Cartan invariants. In the particular case of curves, if $G \subset \mathrm{GL}(N)$ is a matrix Lie group, then the Maurer-Cartan invariants appear as the entries of the classical Frenet-Serret matrix $\mathcal{D} \rho\left(x, u^{(n)}\right) \cdot \rho\left(x, u^{(n)}\right)^{-1},[\mathbf{1 2}, \mathbf{1 4}, \mathbf{2 8}]$.

In practice, the Maurer-Cartan invariants can be directly determined from the recurrence formulae for the phantom differential invariants, as prescribed by the cross-section (3.6), bypassing their theoretical characterization in terms of the Maurer-Cartan forms. Namely, since $\iota\left(Z_{\sigma}\right)=c_{\sigma}$ is constant, for each $i=1, \ldots, p$, the phantom recurrence relations

$$
0=\iota\left[D_{i}\left(Z_{\sigma}\right)\right]+\sum_{k=1}^{p} R_{i}^{\kappa} \iota\left[\mathbf{v}_{\kappa}^{(n)}\left(Z_{\sigma}\right)\right], \quad \sigma=1, \ldots, r,
$$


form a system of $r$ linear equations that, owing to the transversality of the cross-section, can be uniquely solved for the $r$ Maurer-Cartan invariants $R_{i}^{1}, \ldots, R_{i}^{r}$. Substituting the resulting expressions back into the non-phantom recurrence relations leads to a complete system of identities satisfied by the basic differential invariants, $[\mathbf{9}, \mathbf{3 8}]$.

It is worth pointing out that, since the prolonged vector field coefficients (4.2) are polynomials in the jet coordinates $u_{K}^{\beta}$ of order $\# K \geq 1$, their invariantizations are polynomial functions of the fundamental differential invariants $I_{K}^{\beta}$ for $\# K \geq 1$. As a result, the differential invariant algebra is, typically, rational, and are thus amenable to analysis by adaptations of techniques from computational algebra, [8]. The precise requirements are either that the group acts transitively on $M$, or, if intransitive, that, in some coordinate system, its infinitesimal generators $\mathbf{v}_{1}, \ldots, \mathbf{v}_{r}$ depend rationally on the coordinates $z$ on $M$. For such transitive or infinitesimally rational group actions, if the cross-section functions $Z_{1}, \ldots, Z_{r}$ depend rationally on the jet coordinates - we will refer to these as rational cross-sections - then the Maurer-Cartan invariants are rational functions of the basic invariants $\left(H, I^{(n+1)}\right)$, where $n$ is the order of the moving frame. Moreover, all the resulting recurrence formulae depend rationally on the basic differential invariants, justifying the claim.

Example 4.2. The prolonged infinitesimal generators of the planar Euclidean group action on curve jets, as described in Example 3.5, are

$$
\begin{gathered}
\mathbf{v}_{1}=\partial_{x}, \quad \mathbf{v}_{2}=\partial_{u} \\
\mathbf{v}_{3}=-u \partial_{x}+x \partial_{u}+\left(1+u_{x}^{2}\right) \partial_{u_{x}}+3 u_{x} u_{x x} \partial_{u_{x x}}+\left(4 u_{x} u_{x x x}+3 u_{x x}^{2}\right) \partial_{u_{x x x}}+\cdots
\end{gathered}
$$

According to (4.3), the invariant arc length derivative $\mathcal{D}=\iota\left(D_{x}\right)$ of a differential invariant $I=\iota(F)$ is specified by the recurrence relation

$$
\mathcal{D} \iota(F)=\iota\left(D_{x} F\right)+R^{1} \iota\left(\mathbf{v}_{1}(F)\right)+R^{2} \iota\left(\mathbf{v}_{2}(F)\right)+R^{3} \iota\left(\mathbf{v}_{3}(F)\right),
$$

where $R^{\nu}$ are the Maurer-Cartan invariants. To determine their formulas, we write out (4.7) for the three phantom invariants:

$$
\begin{aligned}
& 0=\mathcal{D} \iota(x)=\iota(1)+R^{1} \iota\left(\mathbf{v}_{1}(x)\right)+R^{2} \iota\left(\mathbf{v}_{2}(x)\right)+R^{3} \iota\left(\mathbf{v}_{3}(x)\right)=1+R^{1}, \\
& 0=\mathcal{D} \iota(u)=\iota\left(u_{x}\right)+R^{1} \iota\left(\mathbf{v}_{1}(u)\right)+R^{2} \iota\left(\mathbf{v}_{2}(u)\right)+R^{3} \iota\left(\mathbf{v}_{3}(u)\right)=R^{2}, \\
& 0=\mathcal{D} \iota\left(u_{x}\right)=\iota\left(u_{x x}\right)+R^{1} \iota\left(\mathbf{v}_{1}\left(u_{x}\right)\right)+R^{2} \iota\left(\mathbf{v}_{2}\left(u_{x}\right)\right)+R^{3} \iota\left(\mathbf{v}_{3}\left(u_{x}\right)\right)=\kappa+R^{3} .
\end{aligned}
$$

Solving, we find

$$
R^{1}=-1, \quad R^{2}=0, \quad R^{3}=-\kappa=-I_{2} .
$$

Using these, the general recurrence relation (4.7) becomes

$$
\mathcal{D} \iota(F)=\iota\left(D_{x} F\right)-\iota\left(\mathbf{v}_{1}(F)\right)-\kappa \iota\left(\mathbf{v}_{3}(F)\right) .
$$

In particular, the arc length derivatives of the basic invariants $I_{k}=\iota\left(u_{k}\right)=\iota\left(D_{x}^{k} u\right)$ are given by

$$
\mathcal{D} I_{k}=I_{k+1}-\frac{1}{2} I_{2} \sum_{j=2}^{k-1}\left(\begin{array}{c}
k+1 \\
j
\end{array}\right) I_{j} I_{k-j+1}
$$


of which the first few are

$$
\begin{aligned}
\kappa_{s} & =\mathcal{D} I_{2}=I_{3}, & & \mathcal{D} I_{4}=I_{5}-10 I_{2}^{2} I_{3}, \\
\kappa_{s s} & =\mathcal{D} I_{3}=I_{4}-3 I_{2}^{3}, & & \mathcal{D} I_{5}=I_{6}-15 I_{2}^{2} I_{4}-10 I_{2} I_{3}^{2} .
\end{aligned}
$$

These can be iteratively solved to produce the explicit formulae

$$
\begin{aligned}
\kappa & =I_{2}, & & I_{2}=\kappa, \\
\kappa_{s} & =I_{3}, & & I_{3}=\kappa_{s}, \\
\kappa_{s s} & =I_{4}-3 I_{2}^{3}, & & I_{4}=\kappa_{s s}+3 \kappa^{3}, \\
\kappa_{s s s} & =I_{5}-19 I_{2}^{2} I_{3}, & & I_{5}=\kappa_{s s s}+19 \kappa^{2} \kappa_{s}, \\
\kappa_{s s s s} & =I_{6}-34 I_{2}^{2} I_{4}-48 I_{2} I_{3}^{2}+57 I_{2}^{5}, & & I_{6}=\kappa_{s s s s}+34 \kappa^{2} \kappa_{s s}+48 \kappa \kappa_{s}^{2}+45 \kappa^{5},
\end{aligned}
$$

relating the fundamental normalized and differentiated curvature invariants.

The recurrence relations can be straightforwardly extended to invariant differential forms. Namely, if $\Omega$ is any differential form on $\mathrm{J}^{n}$, then

$$
d \iota(\Omega)=\iota(d \Omega)+\sum_{\kappa=1}^{r} \nu^{\kappa} \wedge \iota\left[\mathbf{v}_{\kappa}^{(n)}(\Omega)\right],
$$

where $\mathbf{v}_{\kappa}^{(n)}(\Omega)$ denotes the Lie derivative of $\Omega$ with respect to the prolonged infinitesimal generator, while $\nu^{\kappa}=\rho^{*} \mu^{\kappa}$ are the pulled-back Maurer-Cartan forms. For our purposes, we only need to look at the case when $\Omega=d x^{i}$ is a basis horizontal form, whereby

$$
d \varpi^{i}=d \iota\left(d x^{i}\right)=\iota\left(d^{2} x^{i}\right)+\sum_{\kappa=1}^{r} \nu^{\kappa} \wedge \iota\left(d \xi_{\kappa}^{i}\right)=\sum_{\kappa=1}^{r} \nu^{\kappa} \wedge \iota\left(d \xi_{\kappa}^{i}\right) .
$$

Ignoring the contact components, and using (3.14), (4.5), we are led to

$$
d_{H} \omega^{i}=\sum_{\kappa=1}^{r} \sum_{j=1}^{p} \sum_{k=1}^{p} R_{j}^{\kappa} \iota\left(D_{k} \xi_{\kappa}^{i}\right) \omega^{j} \wedge \omega^{k}
$$

On the other hand, applying $d_{H}$ to (3.14) and then recalling (2.1), we find

$$
\begin{aligned}
0=d_{H}^{2} F & =\sum_{i=1}^{p}\left[d_{H}\left(\mathcal{D}_{i} F\right) \wedge \omega^{i}+\left(\mathcal{D}_{i} F\right) d_{H} \omega^{i}\right] \\
& =\sum_{j=1}^{p} \sum_{k=1}^{p} \mathcal{D}_{j}\left(\mathcal{D}_{k} F\right) \omega^{j} \wedge \omega^{k}+\sum_{i=1}^{p}\left(\mathcal{D}_{i} F\right) d_{H} \omega^{i} \\
& =\sum_{i=1}^{p} \sum_{j<k} Y_{j k}^{i}\left(\mathcal{D}_{i} F\right) \omega^{j} \wedge \omega^{k}+\sum_{i=1}^{p} \sum_{\kappa=1}^{r} \sum_{j=1}^{p} \sum_{k=1}^{p} R_{j}^{\kappa} \iota\left(D_{k} \xi_{\kappa}^{i}\right)\left(\mathcal{D}_{i} F\right) \omega^{j} \wedge \omega^{k} .
\end{aligned}
$$

Since $F$ is arbitrary, we can equate the individual coefficients of $\left(\mathcal{D}_{i} F\right) \omega^{j} \wedge \omega^{k}$, for $j<k$, to zero, thereby producing explicit formulae for the commutator invariants:

$$
Y_{j k}^{i}=\sum_{\kappa=1}^{r}\left[R_{k}^{\kappa} \iota\left(D_{j} \xi_{\kappa}^{i}\right)-R_{j}^{\kappa} \iota\left(D_{k} \xi_{\kappa}^{i}\right)\right] .
$$




\section{Generating Invariants.}

A set of differential invariants $\mathcal{I}=\left\{I_{1}, \ldots, I_{l}\right\}$ is called generating if, locally, every differential invariant can be expressed as a function of them and their iterated invariant derivatives $\mathcal{D}_{J} I_{\sigma}$. Let us present a few general results in this vein, followed by some specific examples - all consequences of the all-important recurrence relations.

Let

$$
\mathcal{I}^{(n)}=\left\{H^{1}, \ldots, H^{p}\right\} \cup\left\{I_{J}^{\alpha} \mid \alpha=1, \ldots, q, 0 \leq \# J \leq n\right\}
$$

denote the complete set of fundamental differential invariants arising from invariantization of the jet coordinates of order $\leq n$. In particular, assuming we choose a crosssection $K^{n} \subset \mathrm{J}^{n}$ that projects to a cross-section $\pi_{0}^{n}\left(K^{n}\right) \subset M$, then the invariants $\mathcal{I}^{(0)}=\left\{H^{1}, \ldots, H^{p}, I^{1}, \ldots I^{q}\right\}$ are the ordinary invariants for the action on $M$. If $G$ acts transitively on $M$, the latter invariants are all constant (phantom), and hence their inclusion in the following generating systems is superfluous. The first result on generating systems can be found in $[\mathbf{9}]$.

Theorem 5.1. If the moving frame has order $n$, then the set of fundamental differential invariants $\mathcal{I}^{(n+1)}$ of order $n+1$ forms a generating set.

Proof: Since the phantom invariants have order $\leq n$, solving the phantom recurrence relations (4.6) for the Maurer-Cartan invariants implies that the latter have order $\leq n+1$. Let us rewrite the recurrence relation (4.3) for the basic differential invariant $I_{J}^{\alpha}=\iota\left(u_{J}^{\alpha}\right)$ in the form

$$
I_{J, i}^{\alpha}=\mathcal{D}_{i} I_{J}^{\alpha}-\sum_{k=1}^{p} R_{i}^{\kappa} \varphi_{J, \kappa}^{\alpha}\left(H, I^{(k)}\right) .
$$

Consequently, provided $k=\# J \geq n+1$, the left hand side is a basic differential invariant of order $k+1$, while the right hand side depends on differential invariants of order $\leq k$ and their invariant derivatives. A simple reverse induction on $k$ completes the proof. Q.E.D.

As pointed out in $[\mathbf{3 8}, \mathbf{1 4}]$, the smaller generating set claimed in $[\mathbf{9}]$ is only valid when the cross-section has minimal order, in the following sense.

Definition 5.2. A cross-section $K^{n} \subset \mathrm{J}^{n}$ is said to have minimal order if, for all $0 \leq k \leq n$, its projection $K^{k}=\pi_{k}^{n}\left(K^{n}\right)$ forms a cross-section to the prolonged group orbits in $\mathrm{J}^{k}$.

Theorem 5.3. Suppose the differential functions $Z_{1}, \ldots, Z_{r}$ define, as in (3.6), a minimal order cross-section. Let

$$
\mathcal{Z}=\left\{\iota\left(D_{i}\left(Z_{\sigma}\right)\right) \mid i=1, \ldots, p, \sigma=1, \ldots, r\right\}
$$

be the collected invariantizations of their total derivatives. Then $\mathcal{I}^{(0)} \cup \mathcal{Z}$ form a generating set of differential invariants.

Another interesting consequence of the recurrence relations, noticed by Hubert, [13], is that the Maurer-Cartan invariants (4.4) also form a generating set when the action is transitive on $M$. More generally: 
Theorem 5.4. The differential invariants $\mathcal{I}^{(0)} \cup \mathcal{R}$ form a generating set.

Proof: Indeed, by a straightforward induction, the recurrence relations (5.2) imply that, for any $k=\# J>0$, we can rewrite the differential invariants of order $k+1$ in terms of derivatives of those of order $k$ and the Maurer-Cartan invariants.

Q.E.D.

Let us now discuss the problem of finding a minimal generating set of differential invariants. The case of curves, $p=1$, has been well understood for some time. A Lie group is said to act ordinarily, $[\mathbf{3 3}]$, if it acts transitively on $M$, and the maximal dimension of the orbits of its successive prolongations strictly increase until the action becomes locally free; or, in other words, its prolongations do not "pseudo-stabilize", [34]. Almost all transitive Lie group actions are ordinary. For an ordinary action on curves in a $m$-dimensional manifold, there are precisely $q=m-1$ generating differential invariants. Moreover, there are no syzygies among their invariant derivatives. Non-ordinary actions require one additional generator, and a single generating syzygy.

On the other hand, when dealing with submanifolds of dimension $p \geq 2$, I know of no general results on the minimal number of generating differential invariants. And indeed, even in well-studied examples, the conventional wisdom on what differential invariants are required in a minimal generating set is mistaken.

Example 5.5. Consider the standard action of the Euclidean group SE(3) on surfaces $S \subset \mathbb{R}^{3}$ in three-dimensional Euclidean space. The standard local coordinates on the surface jet bundle $\mathrm{J}^{n}=\mathrm{J}^{n}\left(\mathbb{R}^{3}, 2\right)$, for surfaces that can be (locally) identified with graphs $u=f(x, y)$, are $x, y, u, u_{x}, u_{y}, u_{x x}, u_{x y}, u_{y y}, \ldots$, and, in general, $u_{j k}=\partial^{j+k} u / \partial x^{j} \partial y^{k}$ for $j+k \leq n$.

The classical moving frame construction, [12], relies on the coordinate cross-section

$$
x=y=u=u_{x}=u_{y}=u_{x y}=0, \quad u_{x x} \neq u_{y y},
$$

to the locally free prolonged action on $\mathrm{J}^{2}$. The fundamental differential invariants are denoted as $I_{j k}=\iota\left(u_{j k}\right)$. In particular,

$$
\kappa_{1}=I_{20}=\iota\left(u_{x x}\right), \quad \kappa_{2}=I_{02}=\iota\left(u_{y y}\right),
$$

are the principal curvatures; the moving frame is valid provided $\kappa_{1} \neq \kappa_{2}$, meaning that we are at a non-umbilic point. The mean and Gaussian curvature invariants

$$
H=\frac{1}{2}\left(\kappa_{1}+\kappa_{2}\right), \quad K=\kappa_{1} \kappa_{2},
$$

are often used as convenient alternatives, since they eliminate some of the residual discrete ambiguities in the locally equivariant moving frame. (Technically, since $H$ can change its sign under a $180^{\circ}$ rotation that preserves the tangent plane, only $H^{2}$ is a true invariant.) Higher order differential invariants are obtained by differentiation with respect to the Frenet coframe $\omega^{1}=\pi_{H} \iota\left(d x^{1}\right), \omega^{2}=\pi_{H} \iota\left(d x^{2}\right)$, that diagonalizes the first and second fundamental forms of the surface, $[\mathbf{1 2}]$. We let $\mathcal{D}_{1}, \mathcal{D}_{2}$ denote the dual invariant differential operators.

To characterize the full differential invariant algebra, we derive the recurrence relations. Observe that this will not require the explicit formulas for the moving frame or 
the curvature invariants! A basis for the infinitesimal generators for the action on $\mathbb{R}^{3}$ is provided by the 6 vector fields

$$
\begin{gathered}
\mathbf{v}_{1}=-y \partial_{x}+x \partial_{y}, \quad \mathbf{v}_{2}=-u \partial_{x}+x \partial_{u}, \quad \mathbf{v}_{3}=-u \partial_{y}+y \partial_{u}, \\
\mathbf{w}_{1}=\partial_{x}, \quad \mathbf{w}_{2}=\partial_{y}, \quad \mathbf{w}_{3}=\partial_{u} .
\end{gathered}
$$

The recurrence relations (4.3) of order $\geq 1$ have the explicit form

$$
\begin{array}{ll}
\mathcal{D}_{1} I_{j k}=I_{j+1, k}+\sum_{\nu=1}^{3} \varphi_{\nu}^{j k}\left(0,0, I^{(j+k)}\right) R_{1}^{\nu}, & \\
\mathcal{D}_{2} I_{j k}=I_{j, k+1}+\sum_{\nu=1}^{3} \varphi_{\nu}^{j k}\left(0,0, I^{(j+k)}\right) R_{2}^{\nu}, &
\end{array}
$$

Here $R_{1}^{\nu}, R_{2}^{\nu}$, are the Maurer-Cartan invariants associated with the rotational group generator $\mathbf{v}_{\nu}$, while $\varphi_{\nu}^{j k}\left(0,0, I^{(j+k)}\right)=\iota\left[\varphi_{\nu}^{j k}\left(x, y, u^{(j+k)}\right)\right]$ are its invariantized prolongation coefficients, obtained through (4.2). (The translational generators and Maurer-Cartan invariants do not enter into the higher order relations, and so can be ignored.) In particular, the phantom recurrence relations are

$$
\begin{array}{ll}
0=\mathcal{D}_{1} I_{10}=I_{20}+R_{1}^{2}, & 0=\mathcal{D}_{2} I_{10}=R_{2}^{2}, \\
0=\mathcal{D}_{1} I_{01}=R_{1}^{3}, & 0=\mathcal{D}_{2} I_{01}=I_{02}+R_{2}^{3}, \\
0=\mathcal{D}_{1} I_{11}=I_{21}+\left(I_{20}-I_{02}\right) R_{1}^{1}, & 0=\mathcal{D}_{2} I_{11}=I_{12}+\left(I_{20}-I_{02}\right) R_{2}^{1} .
\end{array}
$$

Solving these produces the Maurer-Cartan invariants:

$$
R_{1}^{1}=Y_{2}, \quad R_{1}^{2}=-\kappa_{1}, \quad R_{1}^{3}=0, \quad R_{2}^{1}=-Y_{1}, \quad R_{2}^{2}=0, \quad R_{2}^{3}=-\kappa_{2},
$$

where

$$
Y_{1}=\frac{I_{12}}{I_{20}-I_{02}}=\frac{\mathcal{D}_{1} \kappa_{2}}{\kappa_{1}-\kappa_{2}}, \quad Y_{2}=\frac{I_{21}}{I_{02}-I_{20}}=\frac{\mathcal{D}_{2} \kappa_{1}}{\kappa_{2}-\kappa_{1}},
$$

the second expressions following from the third order recurrence relations

$$
I_{30}=\mathcal{D}_{1} I_{20}=\kappa_{1,1}, \quad I_{21}=\mathcal{D}_{2} I_{20}=\kappa_{1,2}, \quad I_{12}=\mathcal{D}_{1} I_{02}=\kappa_{2,1}, \quad I_{03}=\mathcal{D}_{2} I_{02}=\kappa_{2,2}
$$

Equating the two fourth order recurrence relations

$$
\mathcal{D}_{2} I_{21}+\frac{I_{30} I_{12}-2 I_{12}^{2}}{\kappa_{1}-\kappa_{2}}+\kappa_{1} \kappa_{2}^{2}=I_{22}=\mathcal{D}_{1} I_{12}-\frac{I_{21} I_{03}-2 I_{21}^{2}}{\kappa_{1}-\kappa_{2}}+\kappa_{1}^{2} \kappa_{2},
$$

for $I_{22}=\iota\left(u_{x x y y}\right)$ leads us to the well known Codazzi syzygy

$$
\mathcal{D}_{2}^{2} \kappa_{1}-\mathcal{D}_{1}^{2} \kappa_{2}+\frac{\mathcal{D}_{1} \kappa_{1} \mathcal{D}_{1} \kappa_{2}+\mathcal{D}_{2} \kappa_{1} \mathcal{D}_{2} \kappa_{2}-2\left(\mathcal{D}_{1} \kappa_{2}\right)^{2}-2\left(\mathcal{D}_{2} \kappa_{1}\right)^{2}}{\kappa_{1}-\kappa_{2}}-\kappa_{1} \kappa_{2}\left(\kappa_{1}-\kappa_{2}\right)=0
$$

Using (5.6), we can, in fact, rewrite the Codazzi syzygy in the more succinct form

$$
K=\kappa_{1} \kappa_{2}=-\left(\mathcal{D}_{1}+Y_{1}\right) Y_{1}-\left(\mathcal{D}_{2}+Y_{2}\right) Y_{2}
$$


As noted in [12], the right hand side of (5.9) depends only on the first fundamental form of the surface, and so the Codazzi syzygy (5.9) immediately implies Gauss' Theorema Egregium, that the Gauss curvature is an intrinsic, isometric invariant. Another direct consequence of (5.9) is the Gauss-Bonnet Theorem, [18].

The general commutator formula (4.13) implies that the Maurer-Cartan invariants (5.6) are also the commutator invariants:

$$
\left[\mathcal{D}_{1}, \mathcal{D}_{2}\right]=\mathcal{D}_{1} \mathcal{D}_{2}-\mathcal{D}_{2} \mathcal{D}_{1}=Y_{2} \mathcal{D}_{1}-Y_{1} \mathcal{D}_{2}
$$

Since we are dealing with a second order moving frame, Theorem 5.1 implies that the differential invariant algebra for Euclidean surfaces is generated by the basic differential invariants of order $\leq 3$. However, (5.7) express the third order invariants as invariant derivatives of the principal curvatures $\kappa_{1}, \kappa_{2}$, and hence they, or, equivalently, the Gauss and mean curvatures $H, K$, form a generating system for the differential invariant algebra. This is well known. However, the surprising new result, $[\mathbf{4 0}]$, is that neither is a minimal generating set!

Theorem 5.6. For suitably nondegenerate surfaces, the mean curvature $H$ is a generating differential invariant, i.e., all other Euclidean surface differential invariants can be expressed as functions of $H$ and its invariant derivatives.

Proof: By the preceding remarks, it suffices to express the Gauss curvature $K$ as a function of $H$ and its derivatives. For this, the Codazzi syzygy (5.9) implies that we need only express the commutator invariants $Y_{1}, Y_{2}$, in terms of $H$.

Now, the commutator identity (5.10) can be applied to any differential invariant. In particular,

$$
\begin{aligned}
\mathcal{D}_{1} \mathcal{D}_{2} H-\mathcal{D}_{2} \mathcal{D}_{1} H & =Y_{2} \mathcal{D}_{1} H-Y_{1} \mathcal{D}_{2} H, \\
\mathcal{D}_{1} \mathcal{D}_{2} \mathcal{D}_{j} H-\mathcal{D}_{2} \mathcal{D}_{1} \mathcal{D}_{j} H & =Y_{2} \mathcal{D}_{1} \mathcal{D}_{j} H-Y_{1} \mathcal{D}_{2} \mathcal{D}_{j} H, \quad \text { for } j=1 \text { or } 2 .
\end{aligned}
$$

Thus, provided the nondegeneracy condition

$$
\left(\mathcal{D}_{1} H\right)\left(\mathcal{D}_{2} \mathcal{D}_{j} H\right) \neq\left(\mathcal{D}_{2} H\right)\left(\mathcal{D}_{1} \mathcal{D}_{j} H\right)
$$

holds - which is clearly true for generic surfaces - we can solve the pair of equations (5.11) for the commutator invariants as certain explicit rational combinations of the invariant derivatives of $H$.

Q.E.D.

While most surfaces are non-degenerate, those with constant mean curvature are not, and the preceding argument does not apply. An interesting open question is whether there are other types of degenerate Euclidean surfaces.

A similarly surprising result holds for surfaces in several other classical three-dimensional Klein geometries. First, consider surfaces $S \subset \mathbb{R}^{3}$ under the standard action of the equi-affine group $\mathrm{SA}(3)=\mathrm{SL}(3) \ltimes \mathbb{R}^{3}$ consisting of volume-preserving affine transformations $g \cdot z=A z+b$, where $\operatorname{det} A=1, b \in \mathbb{R}^{3}$. A similar, but more involved argument, based on the recurrence formulae and the commutator trick of (5.11) establishes the following result, $[40]$. 
Theorem 5.7. The algebra of differential invariants for suitably nondegenerate surfaces under the action of the equi-affine group is generated by a single third order differential invariant, known as the Pick invariant, [50], through invariant differentiation.

In $[\mathbf{1 6}, \mathbf{4 2}]$, these results were extended to several other surface geometries.

Theorem 5.8. The differential invariant algebra of a generic surface $S \subset \mathbb{R}^{3}$ under the standard action of

- the centro-equi-affine group $\mathrm{SL}(3)$ is generated by a single second order invariant;

- the conformal group $\mathrm{SO}(4,1)$ is generated by a single third order invariant;

- the projective group PSL(4) is generated by a single fourth order invariant.

Lest the reader be tempted at this juncture to make a general conjecture concerning the differential invariants of surfaces in three-dimensional space, the following elementary example shows that the number of generating invariants can be arbitrarily large.

Example 5.9. Consider the abelian group action

$$
z=(x, y, u) \longmapsto(x+a, y+b, u+p(x, y)),
$$

where $a, b \in \mathbb{R}$ and $p(x, y)$ is an arbitrary polynomial of degree $\leq n$. In this case, for surfaces $u=f(x, y)$, the individual derivatives $u_{j k}$ with $j+k \geq n+1$ form a complete system of independent differential invariants. The invariant differential operators are the usual total derivatives: $\mathcal{D}_{1}=D_{x}, \mathcal{D}_{2}=D_{y}$, which happen to commute. The higher order differential invariants are generated by differentiating the $n+1$ differential invariants $u_{j k}$ of order $n+1=j+k$. Moreover, these invariants clearly form a minimal generating set.

Building on his complete (local) classifications of both finite-dimensional Lie groups and infinite-dimensional Lie pseudo-groups, acting on one- and two-dimensional manifolds, $[\mathbf{2 4}, \mathbf{3 3}]$, Lie, in volume 3 of his monumental treatise on transformation groups, $[\mathbf{2 2}]$, exhibits a large fraction of the three-dimensional classification. He claims to have completed it, but says there is not enough space to present the full details. As far as I know, the remaining calculations have not been found in his notes or personal papers. Later, Amaldi, $[\mathbf{2}, \mathbf{3}]$, lists what he says is the complete classification. More recently, unaware of Amaldi's papers, Komrakov, [19], asserts that such a classification is not possible since one of the branches contains an intractable algebraic problem. Amaldi and Komrakov's competing claims remain to be reconciled, although I suspect that Komrakov is right. Whether or not the Lie-Amaldi classification is complete, it would, nevertheless, be a worthwhile project to systematically analyze the differential invariant algebras of curves and, especially, surfaces under each of the transformation groups appearing in the Amaldi-Lie lists.

Even with the powerful recurrence formulae at our disposal, the general problem of finding and characterizing a minimal set of generating differential invariants remains open. Indeed, I do not know of a verifiable criterion for minimality, except in the trivial case when there is a single generating invariant. Even harder is to devise an algorithm that will produce a minimal generating set. It is worth pointing out that the corresponding problem for polynomial ideals - finding a minimal Hilbert basis - appears to be intractable. However, the special structure of the differential invariant algebra prescribed by the form 
of the recurrence relations gives some reasons for optimism that such a procedure might be possible.

\section{Differential Invariant Algebras of Pseudo-Groups.}

Extending the preceding moving frame theory and algorithms to infinite-dimensional pseudo-groups is more challenging. The primordial complication is that there is, to date, no geometric object that adequately represents an abstract pseudo-group, and so pseudogroups are inextricably tied to their individual actions on geometric spaces. This necessitates a reformulation of the basic definition of an equivariant moving frame map. A second complication is the distinction between a general pseudo-group and the more important, but more restrictive concept of a Lie pseudo-group. The axioms for unrestricted pseudo-groups are straightforwardly adapted from those of local transformation groups, while a Lie pseudo-group must be realized as the space of solutions to a suitable system of differential equations - a non-geometric requirement that complicates the theory, but plays an essential role in the applications.

To avoid technical complications, we will now be restricted to the analytic category. Adapting the constructions to smooth $\left(\mathrm{C}^{\infty}\right)$ pseudo-groups requires some additional care, as noted below.

Definition 6.1. A collection $\mathcal{G}$ of local diffeomorphisms of a manifold $M$ is a pseudogroup if

- $\mathcal{G}$ is closed under restriction: if $U \subset M$ is an open set and $\phi: U \rightarrow M$ is in $\mathcal{G}$, then so is $\phi \mid V$ for all open $V \subset U$.

- Elements of $\mathcal{G}$ can be patched together: if $U_{\nu} \subset M$ are open subsets, $U=\bigcup_{\nu} U_{\nu}$, and $\phi: U \rightarrow M$ is a local diffeomorphism with $\phi \mid U_{\nu} \in \mathcal{G}$ for all $\nu$, then $\phi \in \mathcal{G}$.

- $\mathcal{G}$ is closed under composition: if $\phi: U \rightarrow M$ and $\psi: V \rightarrow M$ are two local diffeomorphisms belonging to $\mathcal{G}$ with $\phi(U) \subset V$, then $\psi \circ \phi \in \mathcal{G}$.

- $\mathcal{G}$ is closed under inverse: if $\phi: U \rightarrow M$ is in $\mathcal{G}$, and $V=\phi(U)$, then $\phi^{-1}: V \rightarrow M$ is also in $\mathcal{G}$.

Note that the second and fourth requirements imply that $\mathcal{G}$ necessarily contains the identity diffeomorphism: $\mathbb{1}(z)=z$ for all $z \in M=\operatorname{dom} \mathbb{1}$. The collection $\mathcal{D}=\mathcal{D}(M)$ of local analytic diffeomorphisms of an analytic manifold $M$ is the simplest example of a pseudo-group. All others are sub-pseudo-groups thereof. Well studied examples include analytic (local) actions of finite-dimensional Lie groups; the pseudo-group of symplectomorphisms, [29]; foliation-preserving transformation groups, [10]; and symmetry pseudogroups of differential equations, $[\mathbf{3 2}]$.

For each $0 \leq n \leq \infty$, let $\mathcal{D}^{(n)} \subset \mathrm{J}^{n}(M, M)$ denote the bundle formed by their $n$-th order jets, which, by the Inverse Function Theorem, is characterized by the non-vanishing of the Jacobian determinant. For $k \geq n$, let $\pi_{n}^{k}: \mathcal{D}^{(k)} \rightarrow \mathcal{D}^{(n)}$ denote the standard projection.

Definition 6.2. A pseudo-group $\mathcal{G} \subset \mathcal{D}$ is called regular of order $n^{\star} \geq 1$ if, for all finite $n \geq n^{\star}$, the pseudo-group jets form an embedded subbundle $\mathcal{G}^{(n)} \subset \mathcal{D}^{(n)}$ and the projection $\pi_{n}^{n+1}: \mathcal{G}^{(n+1)} \rightarrow \mathcal{G}^{(n)}$ is a fibration. 
In the analytic category, the additional Lie requirement on the pseudo-group is encapsulated in the following simplified definition.

Definition 6.3. An analytic pseudo-group $\mathcal{G} \subset \mathcal{D}$ is called a Lie pseudo-group if $\mathcal{G}$ is regular of order $n^{\star} \geq 1$ and, moreover, every local diffeomorphism $\phi \in \mathcal{D}$ satisfying $\mathrm{j}_{n^{\star}} \phi \subset \mathcal{G}^{\left(n^{\star}\right)}$ belongs to the pseudo-group: $\phi \in \mathcal{G}$.

Let us introduce local coordinates $\left(z, Z^{(n)}\right)$ on the diffeomorphism jet bundles $\mathcal{D}^{(n)}$, provided by a system of source coordinates $z=\left(z^{1}, \ldots, z^{m}\right)$ on $M$, target coordinates $Z=\left(Z^{1}, \ldots, Z^{m}\right)$ also on $M$, and associated jet coordinates $Z_{B}^{a}$ representing the partial derivatives $\partial^{k} Z^{a} / \partial z^{b_{1}} \cdots \partial z^{b_{k}}$, with $1 \leq a, b_{1}, \ldots, b_{k} \leq m, 1 \leq k=\# B \leq n$. The source and target projections are given by $\sigma\left(z, Z^{(n)}\right)=z$ and $\tau\left(z, Z^{(n)}\right)=Z$, respectively. In local coordinates, then, the pseudo-group jet subbundle $\mathcal{G}^{\left(n^{\star}\right)} \subset \mathcal{D}^{\left(n^{\star}\right)}$ is characterized by a system of $n^{\star}$-th order partial differential equations

$$
F^{\left(n^{\star}\right)}\left(z, Z^{\left(n^{\star}\right)}\right)=0
$$

known as the determining system for the pseudo-group. The Lie condition says that the local solutions $Z=\phi(z)$ to the determining system are precisely the pseudo-group transformations.

Remark: In most treatments, an additional integrability or involutivity requirement, $[\mathbf{5}, \mathbf{3 3}, \mathbf{2 6}, \mathbf{4 8}]$, is imposed on the determining system. However, as shown in $[\mathbf{1 7}]$ — see also Malgrange, [26] — in the analytic category involutivity is a direct consequence of regularity and local solvability, and thus does not need to be explicitly assumed.

While the Lie condition imposes a technical restriction on the types of pseudo-groups to be considered, in a certain sense it is automatic. Namely, according to [17], any regular pseudo-group $\mathcal{G}$ has a canonical Lie completion $\overline{\mathcal{G}} \supset \mathcal{G}$ - namely, the set of all analytic solutions to the determining system $\mathcal{G}^{\left(n^{\star}\right)}$. Moreover, according to the following result, the Lie completion is indistinguishable as far as its differential invariant algebra and invariant variational bicomplex is concerned.

Theorem 6.4. Any regular non-Lie pseudo-group can be completed to a Lie pseudogroup with the same differential invariants and invariant differential forms.

Let us now extend the equivariant moving frame theory to Lie pseudo-groups. Because we lack an abstract object to represent the pseudo-group, we focus on the pseudo-group jet bundles $\mathcal{G}^{(n)}$ instead. Any convenient system of local coordinates on $\mathcal{G}^{(n)}$ can be used as the pseudo-group parameters of order $n$. If $\mathcal{G}$ is of finite type, and hence represents a finite-dimensional Lie group action, then for $n$ sufficiently large, $\mathcal{G}^{(n)} \rightarrow M$ can be identified with the principle bundle $G \times M$. However, $\mathcal{G}^{(n)}$ does not carry the structure of a group, but rather that of a groupoid, $[\mathbf{2 5}, \mathbf{5 2}]$, whose multiplication is provided by algebraic composition of Taylor series, when defined. In general, then, the multiplication $g^{(n)} \cdot h^{(n)}$ of two elements $g^{(n)}, h^{(n)} \in \mathcal{G}^{(n)}$ is only defined if the source of $g^{(n)}$ matches the target of $h^{(n)}$, i.e., $\sigma\left(g^{(n)}\right)=\tau\left(h^{(n)}\right)$. Consequently, the moving frame theory for pseudo-groups must be formulated in the category of groupoids, not groups. 
Given a Lie pseudo-group action, to define an equivariant moving frame map on the submanifold jet bundle $\mathrm{J}^{n}=\mathrm{J}^{n}(M, p)$, we begin by pulling back the pseudo-group jet bundle $\mathcal{G}^{(n)} \rightarrow M$ via the projection $\pi_{0}^{n}: \mathrm{J}^{n} \rightarrow M$ to produce a bundle $\mathcal{H}^{(n)} \rightarrow \mathrm{J}^{n}$. Local coordinates on $\mathcal{H}^{(n)}$ are given by $\left(z^{(n)}, g^{(n)}\right)$, where $z^{(n)}=\left(x, u^{(n)}\right)$ are the submanifold jet coordinates, while $g^{(n)}$ serve to parametrize the pseudo-group jets. The bundle $\mathcal{H}^{(n)}$ also carries the structure of a groupoid, with source map being projection, $\sigma\left(z^{(n)}, g^{(n)}\right)=z^{(n)}$, while the target map $\tau\left(z^{(n)}, g^{(n)}\right)=g^{(n)} \cdot z^{(n)}$ represents the prolonged action of a pseudogroup diffeomorphism with $n$ jet $g^{(n)}$.

Definition 6.5. A moving frame of order $n$ for a pseudo-group action is an equivariant local section $\rho: \mathrm{J}^{n} \rightarrow \mathcal{H}^{(n)}$.

Equivariance refers to the groupoid structure on $\mathcal{H}^{(n)}$, so that, for a right-equivariant moving frame, $\rho\left(g^{(n)} \cdot z^{(n)}\right)=\rho\left(z^{(n)}\right) \cdot\left(g^{(n)}\right)^{-1}$ when defined. As in the finite-dimensional theory, the existence of a moving frame requires that the pseudo-group action be free and regular. Regularity means, as before, that the pseudo-group orbits form a regular foliation. However, the freeness requirement must be formulated in a different fashion since, for dimensional reasons, any infinite-dimensional pseudo-group that acts on a finitedimensional manifold has necessarily nontrivial isotropy. Instead, we formulate freeness in terms of the induced action of pseudo-group jets.

Definition 6.6. A pseudo-group acts freely on a submanifold jet $z^{(n)} \in \mathrm{J}^{n}$ provided $\tau\left(z^{(n)}, g^{(n)}\right)=z^{(n)}$ if and only if $g^{(n)}=\mathbb{1}^{(n)}$ is the $n$ jet of the identity diffeomorphism.

A foundational result, proved in $[46]$ — see also [45] for the original proof of the locally free version - is the persistence of freeness.

Theorem 6.7. If the pseudo-group $\mathcal{G}$ acts (locally) freely at $z^{(n)}$ then it acts (locally) freely at any $z^{(k)} \in \mathrm{J}^{k}, k>n$, with $\pi_{n}^{k}\left(z^{(k)}\right)=z^{(n)}$.

We say that $\mathcal{G}$ acts eventually freely if it acts freely on an open subset $V^{n} \subset \mathrm{J}^{n}$, and hence on the open subsets $V^{k}=\left(\pi_{n}^{k}\right)^{-1} V^{n} \subset \mathrm{J}^{k}$ for any $k \geq n$. The minimal such $n$ is called the order of freeness, and denoted $n^{\star}$. Freeness serves to bound the dimensions of the pseudo-group jet bundles $G^{(n)}$, and provides a simpler alternative to the Spencer cohomological growth conditions imposed by Kumpera, [21]. We note that, when specialized to a finite-dimensional Lie group action, the pseudo-group Definition 6.6 of freeness is slightly more general than the usual requirement that the isotropy subgroup be trivial.

As in the finite-dimensional version, moving frames are constructed through a normalization procedure based on a choice of cross-section $K^{n} \subset \mathrm{J}^{n}$ to the pseudo-group orbits. Then, as in Theorem 3.3, the group component of the moving frame section $\rho^{(n)}\left(z^{(n)}\right)=\left(z^{(n)}, g^{(n)}\right)$ is determined by the condition that $\tau\left(\rho^{(n)}\left(z^{(n)}\right)\right)=g^{(n)} \cdot z^{(n)} \in K^{n}$. Unlike the finite-dimensional case, though, for an infinite-dimensional pseudo-group, a new cross-section and corresponding moving frame must be selected at each order above the order of freeness. We assume that these cross-sections are compatible, in the sense that $\pi_{n}^{k}\left(K^{k}\right)=K^{n}$ for all $k \geq n \geq n^{\star}$, which implies compatibility of the resulting moving 
frames: $\pi_{n}^{k}\left(\rho\left(z^{(k)}\right)\right)=\rho\left(\pi_{n}^{k}\left(z^{(n)}\right)\right)$. For brevity, we will simply refer to such a compatible sequence of moving frames as a moving frame.

With such a moving frame at hand, the invariantization of functions and differential forms proceeds exactly as before. In particular, the fundamental differential invariants are obtained by invariantizing the jet coordinate functions: $\left(H, I^{(n)}\right)=\iota\left(x, u^{(n)}\right)$. The combinations defining the cross-section $K^{n}$ will be the constant phantom differential invariants, while the remaining basic differential invariants form a complete system of functionally independent differential invariants of order $\leq n$ for the prolonged pseudo-group action on submanifolds. Further, we let $\omega^{i}=\pi_{H} \iota\left(d x^{i}\right), i=1, \ldots, p$, denote the contact-invariant coframe, and $\mathcal{D}_{1}, \ldots, \mathcal{D}_{p}$ the corresponding invariant differential operators, as defined in (3.14). Thus, the differential invariant algebras of eventually free Lie pseudo-groups all have a common structure, shared with the subcategory of finite-dimensional Lie group actions.

Example 6.8. Let $M=\mathbb{R}^{3}$. Consider the Lie pseudo-group

$$
X=f(x), \quad Y=f^{\prime}(x) y+g(x), \quad U=u+\frac{f^{\prime \prime}(x) y+g^{\prime}(x)}{f^{\prime}(x)},
$$

where $f(x)$ is an arbitrary local diffeomorphism of $\mathbb{R}$ while $g(x)$ is an arbitrary analytic function. We are interested in the induced action on surfaces $S \subset M$. To calculate the prolonged pseudo-group transformations, we note that

$$
d_{H} X=f_{x} d x, \quad d_{H} Y=e_{x} d x+f_{x} d y,
$$

where, for convenience, we set

$$
e(x, y)=f^{\prime}(x) y+g(x), \quad \text { and so } \quad e_{y}=f_{x}, \quad f_{y}=0 .
$$

The prolonged pseudo-group transformations are found by successively applying the dual implicit differentiation operators

$$
\mathrm{D}_{X}=\frac{1}{f_{x}} \mathrm{D}_{x}-\frac{e_{x}}{f_{x}^{2}} \mathrm{D}_{y}, \quad \mathrm{D}_{Y}=\frac{1}{f_{x}} \mathrm{D}_{y}
$$

whereby

$$
\begin{gathered}
U=u+\frac{e_{x}}{f_{x}}, \quad U_{X}=\frac{u_{x}}{f_{x}}+\frac{e_{x x}-e_{x} u_{y}}{f_{x}^{2}}-2 \frac{f_{x x} e_{x}}{f_{x}^{3}}, \quad U_{Y}=\frac{u_{y}}{f_{x}}+\frac{f_{x x}}{f_{x}^{2}}, \\
U_{X X}=\frac{u_{x x}}{f_{x}^{2}}+\frac{e_{x x x}-e_{x x} u_{y}-2 e_{x} u_{x y}-f_{x x} u_{x}}{f_{x}^{3}}+ \\
+\frac{e_{x}^{2} u_{y y}+3 e_{x} f_{x x} u_{y}-4 e_{x x} f_{x x}-3 e_{x} f_{x x x}}{f_{x}^{4}}+8 \frac{e_{x} f_{x x}^{2}}{f_{x}^{5}}, \\
U_{X Y}=\frac{u_{x y}}{f_{x}^{2}}+\frac{f_{x x x}-f_{x x} u_{y}-e_{x} u_{y y}}{f_{x}^{3}}-2 \frac{f_{x x}^{2}}{f_{x}^{4}}, \quad U_{Y Y}=\frac{u_{y y}}{f_{x}^{2}},
\end{gathered}
$$

and so on. The pseudo-group does not act freely on $\mathrm{J}^{1}$, but its second prolongation is locally free and locally transitive on the open set $\left\{u_{y y} \neq 0\right\} \subset \mathrm{J}^{2}$. 
To construct a moving frame, we restrict our attention to the case $u_{y y}>0$ and use the following cross-section:

$$
\begin{aligned}
x=y=u=u_{x} & =u_{y}=0, \quad u_{x x}=u_{x y}=0, \quad u_{y y}=1, \\
u_{x^{k}} & =u_{x^{k-1} y}=0, \quad \text { for } \quad k \geq 3,
\end{aligned}
$$

Solving the normalization equations produces the moving frame formulae:

$$
\begin{aligned}
X & =0, & & f=0, \\
Y & =0, & & e=0, \\
U & =0, & & e_{x}=-u f_{x}, \\
U_{Y} & =0, & & f_{x x}=-u_{y} f_{x}, \\
U_{X} & =0, & & e_{x x}=\left(u u_{y}-u_{x}\right) f_{x}, \\
U_{Y Y} & =1, & & f_{x}=\sqrt{u_{y y}}, \\
U_{X Y} & =0, & & f_{x x x}=-\sqrt{u_{y y}}\left(u_{x y}+u u_{y y}-u_{y}^{2}\right), \\
U_{X X} & =0, & & e_{x x x}=-\sqrt{u_{y y}}\left(u_{x x}-u u_{x y}-2 u^{2} u_{y y}-2 u_{x} u_{y}+u u_{y}^{2}\right) .
\end{aligned}
$$

By this stage, we have normalized enough parameters to find the first two fundamental differential invariants of the pseudo-group, namely,

$$
J_{1}=\iota\left(u_{x y y}\right)=\frac{u_{x y y}+u u_{y y y}+2 u_{y} u_{y y}}{u_{y y}^{3 / 2}}, \quad J_{2}=\iota\left(u_{y y y}\right)=\frac{u_{y y y}}{u_{y y}^{3 / 2}} .
$$

Substituting the pseudo-group normalizations into (6.3) fixes the contact-invariant coframe

$$
\omega^{1}=\pi_{H}(\iota(d x))=\sqrt{u_{y y}} d x, \quad \omega^{2}=\pi_{H}(\iota(d y))=\sqrt{u_{y y}}(d y-u d x),
$$

with dual invariant differential operators

$$
\mathcal{D}_{1}=\frac{1}{\sqrt{u_{y y}}}\left(\mathrm{D}_{x}+u \mathrm{D}_{y}\right), \quad \mathcal{D}_{2}=\frac{1}{\sqrt{u_{y y}}} \mathrm{D}_{y} .
$$

As we shall subsequently prove, $J_{1}, J_{2}$ serve as a generating set for the differential invariant algebra. The commutation relation for the invariant differential operators,

$$
\left[\mathcal{D}_{1}, \mathcal{D}_{2}\right]=J_{2} \mathcal{D}_{1}-J_{1} \mathcal{D}_{2}
$$

can be deduced from the recurrence formulae for the contact-invariant coframe, in analogy with the finite-dimensional version (4.13), or simply by direct computation using the explicit formulas (6.9). Finally, there is a single fundamental syzygy among the generating differential invariants,

$$
\mathcal{D}_{1} J_{2}-\mathcal{D}_{2} J_{1}=2,
$$

which can easily be checked directly, but also follows from the recurrence formulae developed below. 
Let us now present the recurrence formulae and resulting structure of the differential invariant algebra of an eventually free Lie pseudo-group action. As argued in [43], the Maurer-Cartan forms for a pseudo-group $\mathcal{G}$ are obtained by restricting the right-invariant contact forms on the infinite diffeomorphism jet bundle to the pseudo-group jet subbundle $\mathcal{G}^{(\infty)} \subset \mathcal{D}^{(\infty)}$. The explicit construction is not required here, but can be found in the aforementioned reference. A basis for the right-invariant contact one-forms is written $\mu_{B}^{a}$ for $a=1, \ldots, m, B=\left(b_{1}, \ldots, b_{k}\right)$, with $1 \leq b_{\nu} \leq m$ and $k=\# B \geq 0$. When restricted to $\mathcal{G}^{(\infty)}$, the resulting one-forms are no longer linearly independent. Remarkably, the induced constraints can be immediately constructed from the infinitesimal generators of the pseudo-group, which are the locally defined vector fields

$$
\mathbf{v}=\sum_{\alpha=1}^{q} \zeta^{a}(z) \frac{\partial}{\partial z^{a}}
$$

whose flows belong to $\mathcal{G}$. The infinitesimal generators are constrained by a linear system of partial differential equations

$$
L^{(n)}\left(z, \zeta^{(n)}\right)=0
$$

where $\zeta^{(n)}=\left(\ldots \zeta_{B}^{a} \ldots\right)$ represents the derivatives (jet coordinates) of the infinitesimal generator coefficients: $\zeta_{B}^{a}=\partial^{k} \zeta^{a} / \partial z^{b_{1}} \cdots \partial z^{b_{k}}, 0 \leq k=\# B \leq n$. This system is obtained by linearizing the pseudo-group's determining system (6.1) at the identity jet, and hence is known as the linearized or infinitesimal determining system. If $\mathcal{G}$ arises as the symmetry group of a system of differential equations, then the linearized determining system (6.13) is the involutive completion of the usual symmetry determining equations obtained via Lie's algorithm, [32].

Theorem 6.9. For each $n \geq 0$, the linear algebraic system

$$
L^{(n)}\left(Z, \mu^{(n)}\right)=0
$$

which is formally obtained from the linearized determining system (6.13) by replacing the source coordinates $z^{a}$ by the corresponding target coordinates $Z^{a}$, and the vector field jet coordinates $\zeta^{(n)}=\left(\ldots \zeta_{B}^{a} \ldots\right)$ by the corresponding right-invariant Maurer-Cartan form $\mu^{(n)}=\left(\ldots \mu_{B}^{a} \ldots\right)$, serves to define the complete set of dependencies among the Maurer-Cartan forms.

Given a moving frame section $\rho: \mathrm{J}^{n} \rightarrow \mathcal{H}^{(n)}$, let $\gamma^{(n)}=\left(\ldots \gamma_{B}^{a} \ldots\right)=\pi_{H} \rho^{*} \mu^{(n)}$ denote the horizontal components of the pulled-back Maurer-Cartan forms. As in the finite-dimensional version, they are linear combinations of the contact-invariant coframe $\omega^{1}, \ldots, \omega^{p}$, whose precise formulas follow directly from the recurrence relations for the phantom differential invariants. In view of Theorem 6.9, the pulled-back Maurer-Cartan forms are subject to the linear relations

$$
L^{(n)}\left(H, I, \gamma^{(n)}\right)=\iota\left[L^{(n)}\left(z, \zeta^{(n)}\right)\right]=0, \quad n \geq 0
$$

obtained by formally invariantizing the linear determining system (6.13), using the convention $\iota\left(\zeta^{(n)}\right)=\gamma^{(n)}$, and where $(H, I)=\iota(x, u)=\iota(z)$. If $\mathcal{G}$ acts transitively on $M$, and we use a minimal order moving frame, so $\left.K^{n} \subset \mathrm{J}^{n}\right|_{z_{0}}$, then the latter are all constant. 
With this in hand, the universal recurrence formula for differential invariants and invariant differential forms of Lie pseudo-groups can be stated.

Theorem 6.10. If $\Omega$ is any differential form on $\mathrm{J}^{n}$, then

$$
d \iota(\Omega)=\iota\left[d \Omega+\mathbf{v}^{(n)}(\Omega)\right]
$$

where the second term on the right hand side denotes the Lie derivative of $\Omega$ with respect to the prolonged vector field $\mathbf{v}^{(n)}$, and ones uses the rule $\iota\left(\zeta_{B}^{a}\right)=\gamma_{B}^{a}$ to invariantize the derivatives of the infinitesimal generator coefficient appearing therein.

Each phantom differential invariant is, by definition, normalized to a constant value, and hence has zero differential. Consequently, the phantom recurrence formulae form a system of linear algebraic equations which, provided $n \geq n^{\star}$, can be uniquely solved for the pulled-back Maurer-Cartan forms $\gamma^{(n)}$. Substituting the resulting expressions into the remaining, non-phantom recurrence formulae leads to a complete system of recurrence relations, which prescribes the structure of the differential invariant algebra for the pseudogroup. As before, if $\mathcal{G}$ acts transitively, or infinitesimally rationally, on $M$, and we choose a coordinate or, more generally, rational cross-section, then the resulting differential invariant algebra is endowed with an entirely rational algebraic recurrence structure.

The constructive proofs of the Basis and Syzygy Theorems for differential invariant algebras of eventually free Lie pseudo-groups, [45], are more subtle than in the finitedimensional Lie group context, and rely on Gröbner basis methods from computational algebra, [8]. There are two important modules associated with the prolonged pseudogroup action. At each $z \in M$, let $\left.\mathcal{I}\right|_{z}$ denote the symbol module, $[\mathbf{5}, \mathbf{4 8}]$, of the linearized determining system (6.13). Integrability implies that $\left.\mathcal{I}\right|_{z}$ forms a submodule of the $\mathbb{R}[t]$ module $\mathcal{T} \simeq \mathbb{R}[t] \otimes \mathbb{R}^{m}$ consisting of real polynomials $\eta(t, T)=\sum_{\alpha=1}^{q} \eta_{a}(t) T^{a}$ depending on $t=\left(t_{1}, \ldots, t_{m}\right)$ and linearly on $T=\left(T^{1}, \ldots, T^{m}\right)$.

Analogously, let $\mathcal{S} \simeq \mathbb{R}[s] \otimes \mathbb{R}^{q}$ denote the $\mathbb{R}[s]$ module consisting of polynomials $\sigma(s, S)=\sum_{\alpha=1}^{q} \sigma_{\alpha}(s) S^{\alpha}$ depending on $s=\left(s_{1}, \ldots, s_{p}\right)$ and linearly on $S=\left(S^{1}, \ldots, S^{q}\right)$. At each submanifold 1 -jet $z^{(1)}=\left(x, u^{(1)}\right)=\left(\ldots x^{i} \ldots u^{\alpha} \ldots u_{i}^{\alpha} \ldots\right) \in \mathrm{J}^{1}$, we define a linear $\operatorname{map} \boldsymbol{\beta}: \mathbb{R}^{m} \times \mathbb{R}^{m} \rightarrow \mathbb{R}^{m}$ by $(s, S)=\boldsymbol{\beta}\left(z^{(1)}, t, T\right)$, with components

$$
\begin{aligned}
s_{i} & =t_{i}+\sum_{\alpha=1}^{q} u_{i}^{\alpha} t_{p+\alpha}, & i & =1, \ldots, p, \\
S^{\alpha} & =T^{p+\alpha}-\sum_{i=1}^{p} u_{i}^{\alpha} T^{i}, & \alpha & =1, \ldots, q .
\end{aligned}
$$

The linear map $\boldsymbol{\beta}$ acts on polynomials via pull-back:

$$
\boldsymbol{\beta}^{*}[\sigma(s, S)]=\sigma\left(\boldsymbol{\beta}\left(z^{(1)}, t, T\right)\right) .
$$

Given $z^{(1)} \in \mathrm{J}^{1}$ with $\pi_{0}^{1}\left(z^{(1)}\right)=z \in M$, the prolonged symbol module $\left.\mathcal{J}\right|_{z^{(1)}} \subset \mathcal{S}$ is defined as the inverse image of the symbol module $\left.\mathcal{I}\right|_{z} \subset \mathcal{T}$ under the pull-back map, so

$$
\left.\mathcal{J}\right|_{z^{(1)}}=\left(\boldsymbol{\beta}^{*}\right)^{-1}\left(\left.\mathcal{I}\right|_{z}\right)=\left\{\sigma\left|\boldsymbol{\beta}^{*}(\sigma) \in \mathcal{I}\right|_{z}\right\}
$$


Invariantization acts coefficient-wise on prolonged symbol polynomials, taking

$$
\sigma\left(x, u^{(1)} ; s, S\right)=\sum_{\alpha=1}^{q} \sum_{\# J \geq 0} h_{\alpha}^{J}\left(x, u^{(1)}\right) s_{J} S^{\alpha},
$$

say, to

$$
\widetilde{\sigma}\left(H, I^{(1)} ; s, S\right)=\iota\left[\sigma\left(x, u^{(1)} ; s, S\right)\right]=\sum_{\alpha=1}^{q} \sum_{\# J \geq 0} h_{\alpha}^{J}\left(H, I^{(1)}\right) s_{J} S^{\alpha} .
$$

We let $\left.\widetilde{\mathcal{J}}\right|_{\left(H, I^{(1)}\right)}=\iota\left(\left.\mathcal{J}\right|_{z^{(1)}}\right)$ denote the invariantized prolonged symbol module, and $\left.\widetilde{\mathcal{J}}^{>n^{\star}}\right|_{\left(H, I^{(1)}\right)}$ the submodule containing those polynomials that have degree $>n^{\star}$ in the $s$ 's. In particular, if $\mathcal{G}$ acts transitively on $\mathrm{J}^{1}$ and we use a minimal order moving frame, then $H, I^{(1)}$ are all constant, and so the invariantized prolonged symbol module is independent of the jet. We identify the invariantized polynomial (6.19) with the differential invariant

$$
I_{\tilde{\sigma}}=\sum_{\alpha=1}^{q} \sum_{\# J \geq 0} h_{\alpha}^{J}\left(H, I^{(1)}\right) I_{J}^{\alpha}
$$

This collection of differential invariants turns out to be much better adapted to the structure of the differential invariant algebra, since their recurrence formulae (6.16) take the form

$$
\mathcal{D}_{i} I_{\tilde{\sigma}}=I_{s_{i} \tilde{\sigma}}+M_{\tilde{\sigma}, i},
$$

in which, when $\operatorname{deg} \widetilde{\sigma}>n^{\star}$, the leading term $I_{s_{i}} \tilde{\sigma}$ is strictly of higher order than the correction term $M_{\tilde{\sigma}, i}$. With this in hand, iteration of (6.21) suffices to establish the Basis Theorem for the differential invariant algebra, $[\mathbf{4 5}]$.

Theorem 6.11. Let $\mathcal{G}$ be a Lie pseudo-group that acts freely an open subset of the submanifold jet bundle at order $n^{\star}$. Then a finite generating system for its differential invariant algebra consists of:

- the differential invariants $I_{\nu}=I_{\sigma_{\nu}}$, where $\sigma_{1}, \ldots, \sigma_{l}$ form a Gröbner basis for the invariantized prolonged symbol submodule $\tilde{\mathcal{J}}^{>n^{\star}}$, and, possibly,

- a finite number of additional differential invariants of order $\leq n^{\star}$.

We are also able to exhibit a finite generating system of differential syzygies. First, owing to the non-commutative nature of the the invariant differential operators, we have the commutator syzygies

$$
\mathcal{D}_{J} I_{\tilde{\sigma}}-\mathcal{D}_{\tilde{J}} I_{\tilde{\sigma}}=M_{\tilde{\sigma}, J}-M_{\tilde{\sigma}, \tilde{J}} \equiv N_{J, \tilde{J}, \tilde{\sigma}}, \quad \text { whenever } \quad \widetilde{J}=\pi(J)
$$

for some permutation $\pi$. Provided $\operatorname{deg} \widetilde{\sigma}>n^{\star}$, the right hand side $N_{J, \tilde{J}, \tilde{\sigma}}$ is of lower order than the terms on the left hand side. Technically, there are an infinite number of algebraically independent commutator syzygies, although they are consequences of the two sided ideal of invariant differential operators that is finitely generated by the original commutator identities (2.1). 
In addition, any algebraic syzygy that is satisfied by the Gröbner basis polynomials in $\left.\widetilde{\mathcal{J}}\right|_{\left(H, I^{(1)}\right)}$ provides an additional differential syzygy amongst the generating invariants. In detail, to each invariantly parametrized polynomial

$$
q\left(H, I^{(1)} ; s\right)=\sum_{J} q_{J}\left(H, I^{(1)}\right) s_{J} \in \mathbb{R}[s]
$$

we associate an invariant differential operator

$$
q\left(H, I^{(1)} ; \mathcal{D}\right)=\sum_{J} q_{J}\left(H, I^{(1)}\right) \mathcal{D}_{J}
$$

where, by convention, we adopt the "normal ordering" that the latter sum ranges over nondecreasing multi-indices $j_{1} \leq j_{2} \leq \cdots \leq j_{k}$. In view of $(6.21)$, whenever $\widetilde{\sigma}\left(H, I^{(1)} ; s, S\right) \in$ $\left.\widetilde{\mathcal{J}}\right|_{\left(H, I^{(1)}\right)}$, we can write

$$
q\left(H, I^{(1)} ; \mathcal{D}\right) I_{\tilde{\sigma}\left(H, I^{(1)} ; s, S\right)}=I_{q\left(H, I^{(1)} ; s\right) \tilde{\sigma}\left(H, I^{(1)} ; s, S\right)}+R_{q, \tilde{\sigma}}
$$

where $R_{q, \tilde{\sigma}}$ has order $<\operatorname{deg} q+\operatorname{deg} \widetilde{\sigma}$. Thus, any algebraic syzygy

$$
\sum_{\nu=1}^{l} q_{\nu}\left(H, I^{(1)}, s\right) \sigma_{\nu}\left(H, I^{(1)} ; s, S\right)=0
$$

among the Gröbner basis polynomials of the invariantized prolonged symbol module induces a syzygy among the generating differential invariants,

$$
\sum_{\nu=1}^{l} q_{\nu}\left(H, I^{(1)}, \mathcal{D}\right) I_{\tilde{\sigma}_{\nu}}=R, \quad \text { where } \quad \operatorname{order} R<\max \left\{\operatorname{deg} q_{\nu}+\operatorname{deg} \widetilde{\sigma}_{\nu}\right\} .
$$

By combining these constituents, we deduce a general, constructive Syzygy Theorem for differential invariant algebras of eventually free Lie pseudo-groups.

Theorem 6.12. Every differential syzygy among the generating differential invariants is a combination of the following:

- the syzygies among the differential invariants of order $\leq n^{\star}$,

- the commutator syzygies,

- syzygies arising from an algebraic syzygy among the Gröbner basis polynomials.

Example 6.13. For the pseudo-group treated in Example 6.8, recall that the order of freeness is $n^{\star}=2$. Since there are no nonconstant first order differential invariants, we can ignore the dependence of the invariantized symbol polynomials, etc., on $H, I^{(1)}=$ const. In view of the cross-section equations (6.5), the prolonged symbol submodule $\mathcal{J}^{>2}$ is spanned by the monomials $s_{1}^{i} s_{2}^{j} S$ for $i+j \geq 3, j \geq 2$. Thus, the Gröbner basis consists of the monomials

$$
\widetilde{\sigma}_{1}=s_{1} s_{2}^{2} S, \quad \widetilde{\sigma}_{2}=s_{2}^{3} S,
$$

whose corresponding differential invariants $J_{1}=I_{1,2}, J_{2}=I_{0,3}$, appear in (6.7). Since there are no low order differential invariants, Theorem 6.11 immediately implies that $J_{1}, J_{2}$ 
generate the differential invariant algebra. Furthermore, there is a single generating syzygy among the Gröbner basis polynomials, namely,

$$
s_{2} \widetilde{\sigma}_{1}-s_{1} \widetilde{\sigma}_{2}=0,
$$

which corresponds to the basic differential syzygy (6.11). Theorem 6.12 implies that the syzygies among the differentiated invariants are all differential consequences of it and the commutation relation (6.10).

Further details and applications of these results can be found in $[\mathbf{7}, \mathbf{4 4}, \mathbf{4 5}, \mathbf{4 9}]$.

Acknowledgments: Thanks to Rob Thompson, Patrick Campbell, and the referees for corrections and comments.

\section{References}

[1] Akivis, M.A., and Rosenfeld, B.A., Élie Cartan (1869-1951), Translations Math. Monographs, vol. 123, American Math. Soc., Providence, R.I., 1993.

[2] Amaldi, U., Contributo all determinazione dei gruppi continui finiti dello spazio ordinario I, Giornale Mat. Battaglini Prog. Studi Univ. Ital. 39 (1901), 273-316.

[3] Amaldi, U., Contributo all determinazione dei gruppi continui finiti dello spazio ordinario II, Giornale Mat. Battaglini Prog. Studi Univ. Ital. 40 (1902), $105-141$.

[4] Anderson, I.M., The Variational Bicomplex, Utah State Technical Report, 1989, http://math.usu.edu/ fg_mp.

[5] Bryant, R.L., Chern, S.-S., Gardner, R.B., Goldschmidt, H.L., and Griffiths, P.A., Exterior Differential Systems, Math. Sci. Res. Inst. Publ., vol. 18, Springer-Verlag, New York, 1991.

[6] Cartan, É., La Méthode du Repère Mobile, la Théorie des Groupes Continus, et les Espaces Généralisés, Exposés de Géométrie, no. 5, Hermann, Paris, 1935.

[7] Cheh, J., Olver, P.J., and Pohjanpelto, J., Algorithms for differential invariants of symmetry groups of differential equations, Found. Comput. Math. 8 (2008), 501-532.

[8] Cox, D., Little, J., and O'Shea, D., Ideals, Varieties, and Algorithms, 2nd ed., Springer-Verlag, New York, 1996.

[9] Fels, M., and Olver, P.J., Moving coframes. II. Regularization and theoretical foundations, Acta Appl. Math. 55 (1999), 127-208.

[10] Fuchs, D.B., Gabrielov, A.M., and Gel'fand, I.M., The Gauss-Bonnet theorem and Atiyah-Patodi-Singer functionals for the characteristic classes of foliations, Topology 15 (1976), 165-188.

[11] Gromov, M., Pseudo holomorphic curves in symplectic manifolds, Inventiones Math. 82 (1985), 307-347.

[12] Guggenheimer, H.W., Differential Geometry, McGraw-Hill, New York, 1963. 
[13] Hubert, E., Generation properties of Maurer-Cartan invariants, preprint, INRIA, 2007.

[14] Hubert, E., Differential invariants of a Lie group action: syzygies on a generating set, J. Symb. Comp. 44 (2009), 382-416.

[15] Hubert, E., and Kogan, I.A., Smooth and algebraic invariants of a group action. Local and global constructions, Found. Comput. Math. 7 (2007), 455-493.

[16] Hubert, E., and Olver, P.J., Differential invariants of conformal and projective surfaces, SIGMA 3 (2007), 097.

[17] Itskov, V., Olver, P.J., and Valiquette, F., Lie completion of pseudo-groups, preprint, University of Minnesota, 2009.

[18] Kogan, I.A., and Olver, P.J., Invariant Euler-Lagrange equations and the invariant variational bicomplex, Acta Appl. Math. 76 (2003), 137-193.

[19] Komrakov, B., Primitive actions and the Sophus Lie problem, in: The Sophus Lie Memorial Conference, O.A. Laudal and B. Jahren, eds., Scandinavian University Press, Oslo, 1994, pp. 187-269.

[20] Kruglikov, B., and Lychagin, V., Invariants of pseudogroup actions: homological methods and finiteness theorem, Int. J. Geom. Methods Mod. Phys. 3 (2006), 1131-1165.

[21] Kumpera, A., Invariants différentiels d'un pseudogroupe de Lie, J. Diff. Geom. 10 (1975), 289-416.

[22] Lie, S., Theorie der Transformationsgruppen, B.G. Teubner, Leipzig, 1888, 1890, 1893.

[23] Lie, S., and Scheffers, G., Vorlesungen über Continuierliche Gruppen mit Geometrischen und Anderen Anwendungen, B.G. Teubner, Leipzig, 1893.

[24] Lie, S., Gruppenregister, in: Gesammelte Abhandlungen, vol. 5, B.G. Teubner, Leipzig, 1924, pp. 767-773.

[25] Mackenzie, K., General Theory of Lie Groupoids and Lie Algebroids, London Math. Soc. Lecture Notes, vol. 213, Cambridge University Press, Cambridge, 2005.

[26] Malgrange, B., Systèmes Différentiels Involutifs, Panoramas et Synthèses No. 19, Societé Mathématique de France, Paris, 2005.

[27] Mansfield, E.L., A Practical Guide to the Invariant Calculus, Cambridge University Press, Cambridge, 2010.

[28] Marí Beffa, G., Projective-type differential invariants and geometric curve evolutions of KdV-type in flat homogeneous manifolds, Ann. Institut Fourier 58 (2008), $1295-1335$.

[29] McDuff, D., and Salamon, D., Introduction to Symplectic Topology, Oxford University Press, Oxford, 1995.

[30] Muñoz, J., Muriel, F.J., and Rodríguez, J., On the finiteness of differential invariants, J. Math. Anal. Appl. 284 (2003), 266-282.

[31] Olver, P.J., Symmetry groups and group invariant solutions of partial differential equations, J. Diff. Geom. 14 (1979), 497-542.

[32] Olver, P.J., Applications of Lie Groups to Differential Equations, Second Edition, Graduate Texts in Mathematics, vol. 107, Springer-Verlag, New York, 1993. 
[33] Olver, P.J., Equivalence, Invariants, and Symmetry, Cambridge University Press, Cambridge, 1995.

[34] Olver, P.J., Pseudo-stabilization of prolonged group actions. I. The order zero case, J. Nonlinear Math. Phys. 4 (1997), 271-277.

[35] Olver, P.J., Moving frames and singularities of prolonged group actions, Selecta Math. 6 (2000), 41-77.

[36] Olver, P.J., Joint invariant signatures, Found. Comput. Math. 1 (2001), 3-67.

[37] Olver, P.J., Geometric foundations of numerical algorithms and symmetry, Appl. Alg. Engin. Commun. Comput. 11 (2001), 417-436.

[38] Olver, P.J., Generating differential invariants, J. Math. Anal. Appl. 333 (2007), 450-471.

[39] Olver, P.J., Invariant submanifold flows, J. Phys. A 41 (2008), 344017.

[40] Olver, P.J., Differential invariants of surfaces, Diff. Geom. Appl. 27 (2009), 230-239.

[41] Olver, P.J., Lectures on moving frames, in: Symmetries and Integrability of Difference Equations, D. Levi, P. Olver, Z. Thomova and P. Winternitz, eds., Cambridge University Press, Cambridge, to appear.

[42] Olver, P.J., Moving frames and differential invariants in centro-affine geometry, Lobachevsky J. Math. 31 (2010), 77-89.

[43] Olver, P.J., and Pohjanpelto, J., Maurer-Cartan forms and the structure of Lie pseudo-groups, Selecta Math. 11 (2005), 99-126.

[44] Olver, P.J., and Pohjanpelto, J., Moving frames for Lie pseudo-groups, Canadian J. Math. 60 (2008), 1336-1386.

[45] Olver, P.J., and Pohjanpelto, J., Differential invariant algebras of Lie pseudo-groups, Adv. Math. 222 (2009), 1746-1792.

[46] Olver, P.J., and Pohjanpelto, J., Persistence of freeness for pseudo-group actions, preprint, University of Minnesota, 2009.

[47] Ovsiannikov, L.V., Group Analysis of Differential Equations, Academic Press, New York, 1982.

[48] Seiler, W.M., Involution: The Formal Theory of Differential Equations and its Applications in Computer Algebra, Algorithms and Computation in Mathematics, vol. 24, Springer-Verlag, New York, 2010.

[49] Shemyakova, E., and Mansfield, E.L., Moving frames for Laplace invariants, in: Proceedings ISSAC2008, D. Jeffrey, ed., ACM, New York, 2008, pp. 295-302.

[50] Spivak, M., A Comprehensive Introduction to Differential Geometry, vol. 3, Third Ed., Publish or Perish, Inc., Houston, TX, 1999.

[51] Tresse, A., Sur les invariants différentiels des groupes continus de transformations, Acta Math. 18 (1894), 1-88.

[52] Weinstein, A., Groupoids: unifying internal and external symmetry. A tour through some examples, Notices Amer. Math. Soc. 43 (1996), 744-752. 